Revue

Revue de l'histoire des religions

del'histoire des religions

$4 \mid 2007$

Varia

\title{
Le serpent guérisseur et l'origine de la gnose ophite
}

The healing serpent and the origin of Ophite Gnosticism

\section{Annarita Magri}

\section{OpenEdition}

Journals

Édition électronique

URL : http://journals.openedition.org/rhr/5351

DOI : $10.4000 /$ rhr.535

ISSN : 2105-2573

Éditeur

Armand Colin

Édition imprimée

Date de publication : 1 décembre 2007

Pagination : 395-434

ISBN : 978-2200-92335-8

ISSN : 0035-1423

\section{Référence électronique}

Annarita Magri, "Le serpent guérisseur et l'origine de la gnose ophite », Revue de l'histoire des religions [En ligne], 4 | 2007, mis en ligne le 01 décembre 2010, consulté le 21 décembre 2020. URL : http:// journals.openedition.org/rhr/5351; DOI : https://doi.org/10.4000/rhr.5351

Tous droits réservés 


\section{ANNARITA MAGRI \\ Université de Fribourg (Suisse) \\ Le serpent guérisseur et l'origine de la gnose ophite}

La branche de la gnose ophite s'enracine probablement dans la secte des Pérates, qui se situe au milieu du II siècle de notre ère et dont parle l'Elenchos 5, 12-18, ouvrage attribué a Hippolyte de Rome. Ce groupe est, avec les Naassènes, le seul à adorer le serpent, considéré comme la manifestation du Logos. Cette équivalence Logos = serpent est tirée à son tour de l'exégèse de Nb. 21, 8-10 (le serpent d'airain). Cette étude s'efforce de retracer le noyau fondamental du récit des hérésiologues sur les Ophites et de le comparer à celui consacré aux Pérates; parallèlement, il sera démontré que le II siècle est l'époque idéale pour y situer le début de l'ophitisme, car c'est à cette époque que l'image du serpent atteignit l'acmé de sa diffusion grâce aux cultes à mystères et, surtout, à la vénération pour Asclépios. Les Pérates eux-mêmes sont issus d'un mélange syncrétiste entre christianisme et culte d'Asclépios.

\section{The healing serpent and the origin of Ophite Gnosticism}

The branch of the Ophite gnosticism takes its origin likely with the sect of the Perates, which is dated around the half of the II ${ }^{\text {nd }}$ century C.E. and which is described by Elenchos 5, 12-18, a work attributed to Hippolytus of Rome. This group is, together with the Naassenes, the only one that actually adores the serpent, which is considered as the epiphany of the Logos. This equivalence Logos = serpent is taken from the exegetical interpretation of Num. 21, 8-10 (the bronze serpent). This study tries to recover the basic chore of the heresiologists' reports about the Ophites and to compare it with the one concerning the Perates. It also shows that the II ${ }^{\text {nd }}$ century was ideal for the beginning of the Ophite Gnosticism: in fact, during this period, the image of the serpent reached the peak of its diffusion, thanks to the mystery cults and to the adoration of Asclepius. The Perates, in fact, take origin from a syncretistical mix of Christianism and Asclepius' cult. 
Zu meiner lieben deutschen Freundin Frau Annelies.

Parmi les hérésies les plus surprenantes regroupées sous l'étiquette de gnostiques figure la branche des Ophites, censés honorer le serpent ${ }^{1}$. À première vue, il est étonnant qu'un groupe probablement influencé par la culture judéo-chrétienne pût vénérer un animal que celle-ci associait couramment à rien moins que le diable ${ }^{2}$, mais, à ma connaissance, personne ne s'est soucié de faire une analyse approfondie du contexte d'où pouvait découler une pareille valorisation ${ }^{3}$. Tel sera le propos de cette contribution : un travail de comparaison

1. Clément d'Alexandrie, Stromates 7,17,108, 2.

2. L'identification du serpent avec le diable procède évidemment de la scène de Gen. 3; Sg. 2, 14 est le premier texte à la poser explicitement, mais il ne faut pas oublier les équivalences entre reptile et diable présupposées ensuite par les targumim, comme Targum Ps. Jonathan 3,6 et 4,1. Sur la valeur du serpent dans la culture juive et chrétienne, voir, par exemple, Werner Foerster, "Ophis », dans Gerhard Kittel-Gerhard Friedrich édd., Grande Lessico del Nuovo Testamento (trad.it), vol. 9, Paideia, Brescia, 1974 (éd. originale allemande, Stuttgart, 1954), 23-66.

3. On ne regrettera jamais assez la disproportion entre l'imposante bibliographie sur les problèmes généraux du gnosticisme, comme l'origine de ce mouvement, et la pénurie d'études ciblées sur certaines hérésies. En fait, les titres concernant les Ophites sont assez réduits et réunissent de nombreux articles d'encyclopédies: voir, par exemple, Hans Leisegang, La Gnose (trad. fr.), Paris, Payot, $1971^{2}$ (édition originale allemande 1924), 81-129; Günther Bornkamm, «Ophiten », dans Paulys Realencyclopädie der classischen Altertumswissenschaft (neue Bearbeitung von G. Wissowa), vol. 18,1, Stuttgart, 1939, 654-58 ; Andrew J. Welburn, «Reconstructing the Ophite diagram », Novum Testamentum 23 (1981), 261-287 ; Jean-Daniel Kaestli, « L'interprétation du serpent de Genèse 3 dans quelques textes gnostiques et la question de la gnose "Ophite" ", dans Julien Ries et alii éd., Gnosticisme et monde hellénistique. Actes du Colloque de Louvain-la-Neuve (11-14 mars 1980) (Publications de l'Institut Orientaliste de Louvain 27), Louvain-la-Neuve, Université Catholique de Louvain-Institut Orientaliste, 1982, 116-30 ; Claudio Gianotto, « OfitiNaasseni », dans Angelo Di Berardino éd., Dizionario Patristico e di Antichità cristiane, vol. II, Casale Monferrato, Marietti, 1984, 2458-60 (cf. aussi l'édition française publiée par F. Vial dans le cadre du Cerf, Paris, 1990, vol. II, 1807-8) ; Deirdre J. Good, « Naassenes, Ophites », dans Everett Ferguson et alii éd., Encyclopaedia of Early Christianity, New York-Londre: Garland, 1990, 635-36 ; Antonio Orbe, «Apuntes sobre el pecado original gnóstico » et «El bautismo de Jesús entre los Ofitas y los Valentinianos », dans Estudios sobre la teología cristiana primitiva (Fuentes Patrísticas Estudios 1) Madrid-Rome, Ciudad Nueva-Pontificia Università Gregoriana, 1994, 271-85 et 441-90. 
historique entre l'ophitisme et la réalité socioculturelle de l'antiquité tardive peut amener à expliquer l'origine de ce phénomène religieux.

\section{LES SOURCES SUR LA GNOSE OPHITE}

Les hérésiologues nous ont livré plusieurs résumés sur la gnose ophite, parfois contradictoires entre eux, souvent répétitifs : le premier est celui tracé par Irénée de Lyon en Haer. I, 30,1-14. Un second, différent, en Haer. 1,30,15, est une courte notice qui présente des gnostiques anonymes, mais clairement intéressés au serpent. Puis nous disposons d'un autre passage bref, Ps.Tertullien, Haer. 2,1-4, du long récit d'Épiphane, Haer. 37 et des résumés de Philastre, Haer. 1 et Théodoret, Haer. 1,14. Dans sa polémique avec Celse, Origène nous livre un long passage axé sur le célèbre « diagramme des Ophites », en Cels. 6, 24-38. Enfin, il y a surtout le Ve livre de l'Élenchos attribué à Hippolyte, tout entier consacré, selon les intentions de l'auteur, aux gnostiques adorateurs du serpent. Dans la réalité, les hérésies regroupées dans ce livre montrent une considération très variable pour le reptile : dans cet article, on fera surtout mention des deux premiers groupes, les Naassènes (ib. 5, 7-11) et les Pérates (ib. 5,12-18), les seuls à manifester une véritable vénération pour cet animal. Les Naassènes, selon Élenchos 5, 7, dériveraient même leur nom de l'hébreu nahas, « serpent ${ }^{4}$.

\section{LE POINT de VUE de JEAN-DANiel KaESTLI}

Dans son précieux article sur l'ophitisme, Jean-Daniel Kaestli remarquait justement que, parmi tous ces candidats, les uniques,

4. Plusieurs écrits de Nag Hammadi, comme l'Origine du monde, l'Hypostase des Archontes et l'Apocryphe de Jean ont été soupçonnés d'appartenir à la branche ophite et Kaestli en tient compte dans son article : «L'interprétation du serpent de Genèse 3... ». Pour le moment je ne les prends pas en considération, en raison des nombreux problèmes posés par la bibliothèque de Nag Hammadi. Mais il est clair que les résultats de cette étude pourraient donner des pistes nouvelles aussi dans ce domaine. 
véritables « adorateurs » du serpent sont les Pérates et les Naassènes, et les Pérates plus encore que les Naassènes ${ }^{5}$. Dans les références à Gn. 3 des écrits de Nag Hammadi (Origine du monde, Hypostase des Archontes et Apocryphe de Jean), le savant identifiait une tendance « docétique »: le serpent y était donc habité temporairement par Sophia ${ }^{6}$ afin que les protoplastes puissent transgresser la prohibition imposée par le Démiurge ; mais cette identification temporaire avec Sophia n'a rien à voir avec une véritable vénération du serpent. Le chercheur inclut aussi dans cet horizon les témoignages d'Irénée et affirme :

«... Plus un texte est marqué par l'influence du christianisme, plus la figure du serpent est nettement séparée de celle de l'instructrice divine de Gen. 3 et plus elle est clairement associée à l'origine du mal ${ }^{7}$. »

Les uniques véritables adorateurs du serpent seraient ainsi, à côté des Naassènes, les Pérates. On aurait donc une première phase gnostique «a-chrétienne » de valorisation du serpent, suivie par une progressive christianisation des groupes et, en parallèle, « démonisation $\gg$ du reptile.

L'article de Kaestli est très important, en premier lieu parce qu'il isole les véritables adorateurs du serpent ; de plus, il souligne de façon très appropriée que l'importance de cette image parmi les Pérates est due à l'exégèse de l'épisode du serpent d'airain, en Nombres 21, 8 et Jean 3,14. En deuxième lieu, il essaie de retracer un parcours cohérent du motif. En revanche, il n'aborde pas la question de sa genèse, ni des relations génétiques parmi les différents groupes décrits. En fait, même si l'influence judéo-chrétienne a sûrement conduit à démoniser cet animal, on peut démontrer que le développement de l'ophitisme et de la vénération du serpent n'est pas étranger au christianisme même, comme le montre l'exégèse de $\mathrm{Nb}$. 21, 8 par les Pérates. C'est donc à partir de là qu'il faut reprendre la question.

5. Jean-Daniel Kaestli, «L'interprétation du serpent de Genèse 3... », 128-30.

6. Ou Prounikos ou tout autre éon féminin porteur de l'étincelle de sagesse pneumatique.

7. Ibid., cit. 126. 


\section{Le témoignage des Pérates sur le Serpent : Nb. 21, 8 et JN. 3,14.}

Comme nous l'avons déjà remarqué, le dossier exégétique des Pérates (cf. Hipp. El. 5, 16, 6-8) ${ }^{8}$ est axé sur l'épisode de $N b .21,8$ dans la perspective de Jn. 3,14 : le serpent d'airain élevé sur la hampe par Moïse n'est rien d'autre que le Logos. Mais là où le Quatrième Évangile évoque une typologie, les gnostiques songent plutôt à une véritable incarnation du Logos, précédant l'apparition du Christ sur terre. Le texte opère aussi une distinction entre le « serpent véritable », le Logos, et les faux serpents, ceux qui mordaient les Hébreux au désert : ils seraient ainsi les « dieux de la destruction », les astres ou archontes planétaires. En accord avec une conception très répandue au II $^{\mathrm{e}}$ siècle, l'ordre astrologique emprisonne donc l'homme dans la nécessité et le destin, dont la génération est l'expression la plus accomplie. Pour cela, le Logos-serpent sauve les hommes de l'ordre d'ici-bas : il protège ceux qui se sont éloignés du corps et de ce monde - allégorisé par l'Égypte - et il les ramène au salut. Cette construction se fonde, en fait, sur une allégorie de l'Exode, dont on trouvera les traces jusqu'à Origène et qui est implicite dans le nom même de la secte : Pérates, « ceux qui traversent » (la Mer Rouge, évidemment) ${ }^{9}$.

La citation exacte du Prologue de Jn. 1,1-4 et les nombreuses allusions au texte johannique présupposent la rédaction finale de celui-ci, traditionnellement datée à la fin du I $^{\mathrm{er}}$ siècle de notre ère ${ }^{10}$, tandis que l'Élenchos est datable du début du $\mathrm{III}^{\mathrm{e}}$ siècle $^{11}$. L'hérésiologue laisse entendre que la secte est assez ancienne (cf. ib. 5, 12,1).

8. Pour le texte, voir Miroslav Marcovich éd., Refutatio omnium haeresium (Patristische Texte und Studien 25), Berlin-New York, de Gruyter, 1986, 183, même si cette édition reconstruit souvent l'écrit de façon arbitraire.

9. Sur l'histoire de ce concept, voir mon étude Il nome dei Perati, à paraître dans Orpheus 2006.

10. Claudio Moreschini-Enrico Norelli, Storia della letteratura cristiana antica. I. Da Paolo all'età costantiniana, Brescia, Morcelliana, 1995, 131-132. L'ouvrage (au moins le premier volume) a été traduit et enrichi en français : cf. Claudio Moreschini-Enrico Norelli, Histoire de la littérature chrétienne antique grecque et latine, Genève, Labor et fides, 2000.

11. Claudio Moreschini-Enrico Norelli, Storia della letteratura cristiana antica..., 340-341. 
Donc, le groupe doit être situé sans aucun doute dans le cours du II $^{\mathrm{e}}$ siècle ; la comparaison avec Justin, qui, nous allons le voir, utilise les mêmes testimonia aux alentours du 160, nous permet de préférer une datation vers le milieu du siècle.

En fait, le noyau du dossier exégétique de $E l .5,16$ consiste dans une liste de testimonia du bois et de la croix, empruntés à la Grande Église $^{12}$. À côté de $N b$. 6-8 et de $J n .3,14$, on peut y retrouver une référence au bâton de Moïse, que le prophète transforma miraculeusement en serpent (cf. Ex. 4, 2-3 et 17,7-15) et qui fut le moyen du passage de la Mer Rouge (cf. Ex. 14,6); on peut y repérer une allusion aux arbres de l'Eden (cf. Gn. 2, 8-10), tandis que El. 5, 14 laisse entrevoir une allusion à l'épisode des douze sources d'Elim (cf. Ex. 15, 22-25) ; El. 5, 17, par contre, utilise le rare Gn. 30, 37-39, où le patriarche Jacob fait multiplier son troupeau bigarré en l'exposant à la vue des branches d'arbres bigarrées. Ce dernier passage biblique est très important, car il est rarement cité dans la littérature chrétienne ancienne.

Ces testimonia chrétiens tournent autour des images du serpent et du bâton ou baguette (en grec, rhabdos) : le bâton, comme tout objet de bois, était associé par les premiers chrétiens à la croix et au Christ. Surtout le motif du serpent d'airain possédait, à partir de l'Évangile de Jean, une profonde valeur christologique : il fait ainsi son apparition dans plusieurs listes de testimonia ligni, par exemple, Barn. 12, 5, Just. IApol. 60, 3 et Dial. 91, 4, ib. 94,1, ib. 112,1-2 et 131, 4, Tert. Iud. 10,10, Marc. 3, 18, 7 et Idol. 5, 3-4, Cipr. Test. 2, 20. Parmi les écrits gnostiques, on doit citer le passage analogue de Test. Ver. 48, 19-49, 10, où le serpent est identifié au Christ ${ }^{13}$. De plus, de nombreuses références bibliques du dossier des Pérates apparaissent dans Just. Dial. 86, 2, un morceau sûrement fondé sur les testimonia ligni ; le serpent d'airain est nommé seulement au paragraphe 91,

12. Voir ma contribution «L'esegesi della setta ofitica dei Perati. Analisi di Ippolito, Haer. V, $16 »$, Apocrypha 14 (2003), 193-223.

13. Voir Annie et Jean-Pierre Mahé éd., Le Témoignage véritable (NH IX, 3). Gnose et martyre (Bibliothèque copte de Nag Hammadi, section « Textes » 23), Québec, Presses de l'Université Laval-Louvain/Paris, Peeters, 1996, 114-17; les Pérates sont mentionnés dans le commentaire, 195, note 162. 
mais celui-ci, après une digression, découle de la même liste des testimonia qui inspirait le $86^{14}$.

On peut donc affirmer que les Pérates ont puisé à des testimonia sur la croix très semblables à ceux utilisés par Justin : mais, par rapport au Dialogue avec Tryphon, les gnostiques semblent avoir privilégié l'image du serpent et celle du bâton (en fait, celui de Moïse avait la tendance à se transformer en serpent). On remarque l'absence d'autres passages presque omniprésents dans les testimonia sur la croix, surtout celui de la bataille avec Amaleq (cf. Ex.17, 8-13), épisode couramment associé au serpent d'airain : Josué remporta la victoire grâce à l'attitude de prière, avec les bras étendus, de Moïse sur la montagne. En tout cas, l'équivalence entre Logos et serpent (ou rhabdos) est clairement présupposée : ici on a vraiment un groupe gnostique pour lequel serpent $=$ Logos. J'aimerais souligner que même les Naassènes ne posent pas ainsi explicitement cette identité. En fait, si on oublie l'étymologie proposée pour le nom du groupe, le reptile disparaît dans le reste de la notice de l'Élenchos : quelquefois on a même du mal à comprendre la raison de l'importance de cet animal pour ces gnostiques.

Il faut maintenant comparer les Pérates avec les autres Ophites.

\section{LES AUTRES OPHITES}

Pour ce qui concerne les autres notices sur les Ophites, je me borne à donner ici des indications rapides, mais on en tirera déjà des conclusions provisoires.

14. Cette connexion a été démontrée par Pierre Prigent, Justin et l'Ancien Testament. L'argumentation scripturaire du traité de Justin contre toutes les hérésies comme source principale du Dialogue avec Tryphon et de la première Apologie, Paris, Gabalda, 1964, 194-202 ; l'ouvrage s'occupe aussi des testimonia crucis, pour lesquels voir Oskar Skarsaune, The Proof from Prophecy. A Study in Justin Martyr's Proof-Text Tradition: Text-Type, Provenance, Theological Profile (Suppl. Novum Testamentum 56), Leyde, E. J. Brill, 1987, particulièrement 215 et 374-378; Martin Albl, « And Scripture cannot be broken ». The Form and Function of the Early Christian Testimonia Collections (Suppl. Novum Testamentum 96), Leyde-Boston-Cologne, Brill, 1999, 101-106 et 155-157. 
Il est préférable de laisser de côté Irénée Haer. 1,30,1-14 : son évaluation très négative du serpent, qui est simplement utilisé par Prounikos comme canal de communication avec Adam et Ève, puis devient la source de tout mal, semble éloigner ce groupe des Pérates. Kaestli a raison d'affirmer que la vision chrétienne du serpent a pesé lourdement sur cette perspective.

Toutefois, ce long passage, une véritable histoire du salut du point de vue gnostique, contient la version la plus accomplie du mythe de Prounikos : l'éon féminin le plus extérieur, plongé dans les eaux d'en bas et dans la matière, n'est plus capable de remonter, raison pour laquelle il se libère de la matière acquise et donne ainsi origine au ciel et au Démiurge. Ce dernier génère sept fils, les archontes planétaires, qui, à leur tour, essaient de modeler l'homme. Mais cette créature est incapable, sans l'étincelle d'en haut, de se tenir debout : le mythe se présente donc dans des termes semblables à Origine $d u$ monde 114, 29-116, 5, Hypostase des archontes 87, 23-88, 17 et à la version des Naassènes (cf. El. 5, 7,3-8). Dans ce cadre, le serpent est plutôt une image serpentiforme, le Nun, conçue par le Démiurge Ialdabaoth : il est à l'origine de tout mal, mais Prounikos se sert de lui pour rendre possible le péché de l'Éden, c'est-à-dire, d'après l'interprétation gnostique, l'accès des protoplastes à la gnose supérieure. Après cette « utilisation », le Démiurge jette Nun du ciel.

Parmi les autres notices, Irénée Haer. 1,30,15 offre un résumé très concis sur une hérésie anonyme selon laquelle Sophia aurait assumé la forme du serpent (Quidam enim ipsam Sophiam Serpentem factam dicunt) : elle réussit ainsi à communiquer la véritable sagesse aux hommes grâce à la transgression de l'Eden. Kaestli a raison de souligner le « docétisme » fondamental de cette conception; mais on pourrait songer aussi à une incarnation de Sophia dans le serpent, d'une façon analogue à ce qui arrive au Logos des Pérates, l'infinitif factam esse demeurant ambigu. En tout cas, si l'ophitisme avait débuté comme adoration authentique de cet animal, ce docétisme pourrait être dû à une atténuation de l'aperçu positif original.

Lipsius faisait dériver les renseignements du Ps.Tertullien, de Philastre et d'Épiphane du Syntagma d'Hippolyte, aujourd'hui 
perdu $^{15}$ : dans le Pseudo-Tertullien on trouve, pour la première fois après les Pérates, une réflexion axée sur le serpent d'airain, qui aurait même donné aux hommes la connaissance du bien et du mal (une claire allusion à $G n$. 3). L'auteur ajoute que les Ophites en question utilisaient le serpent pour consacrer l'Eucharistie, ce qui est tout à fait compatible avec l'équation Logos = serpent.

Le reste du bref passage du Pseudo-Tertullien parle du Démiurge, qui donna origine à de nombreux éons : cela pourrait correspondre à El. 5,14 (Pérates). En outre, le rédacteur ajoute le mythe de la création de l'homme dans les mêmes termes qu'Irénée, Haer. 1, 30, 6 et les Naassènes. Les pages sur les Pérates n'en portent aucune trace : or, on ne peut savoir si le Ps. Tertullien ignore le mythe de Prounikos ou non, car son résumé est bien court. S'il partage ses sources avec Épiphane, il est bien possible que sa version de la création de l'homme présuppose le mythe de Prounikos, car les deux sont strictement liés. Dans l'Elenchos, par contre, les Pérates sont immédiatement précédés par les Naassènes, qui décrivent la création de l'homme dans des termes semblables. On pourrait donc supposer une confluence des sources et des sectes, car les Ophites d'Irénée ne connaissent pas l'importance du serpent d'airain, ni une véritable identification Logos = serpent. En revanche, le Ps.Tertullien semble faire référence à un groupe analogue à celui des Pérates, mais il y mêle un autre, proche des Ophites d'Irénée. On pourrait en tout cas proposer l'hypothèse que le Ps.Tertullien décrive les Pérates sous le nom d'Ophites.

Je ne m'occupe pas ici de la description du célèbre « diagramme des Ophites » donnée par Origène : cela requiert une analyse très détaillée tant des possibles reconstructions du dessin que de la conception planétaire qui en découle. De plus, cette reconstruction demande une comparaison avec la liste des éons, El. 5,14. En tout cas, Origène nous livre une information d'importance capitale : selon Cels. 6, 28, le fondateur des Ophites ne serait autre qu'Euphrate. On sait très bien qu'un des deux fondateurs des Pérates est Euphrate

15. Claudio Moreschini-Enrico Norelli, Storia della letteratura cristiana antica greca e latina..., 355-356. L'ouvrage de Lipsius n'est pas indiqué dans la bibliographie. 
le Pératique (cf. El. 5,12-13) : il est donc possible que les Pérates soient le groupe fondateur de l'ophitisme. Birger Pearson avait déjà eu cette idée ${ }^{16}$. Mais avant de formuler cette hypothèse d'une façon plus complète, j'aimerais dire quelques mots sur la notice d'Épiphane :

1. La secte des Ophites serait fort ancienne et aurait eu des contacts avec le Nicolas blâmé par l'Apocalypse (cf. Ap. 2, 6 et 15).

2. Les Ophites vénèrent le serpent comme dieu et principe de sagesse (référence implicite au péché de l'Eden).

3. Mythe de Prounikos, naissance de Ialdabaoth, génération par lui des sept archontes, création de l'homme : par hargne contre Prounikos et l'homme, qui avait reçu de l'éon féminin l'étincelle spirituelle, Ialdabaoth réagit en envoyant contre lui son fils, le Serpent, qui trompe Ève. La définition de ce Serpent coïncide avec celle donnée par Ir. Haer. 1, 30, 5.

4. Ils ajoutent que nos intestins ont la forme du serpent (= Irénée, Haer. 1, 30, 15).

5. Le serpent apporte la connaissance aux hommes dans l'Eden et est rejeté par Ialdabaoth.

6. Ils consacrent l'Eucharistie en faisant sortir un serpent de sa kyste et en le faisant s'enrouler autour des pains ; ils l'embrassent aussi sur la bouche.

7. Ils introduisent le serpent d'airain, à côté de $M t$. 10,16, comme preuve d'un enseignement positif sur le serpent.

Il faut remarquer la contradiction sur le rôle du serpent dans le péché de l'Eden : présenté d'un côté comme garant de la gnose, il est dit aussi avoir « trompé » Ève par ordre de Ialdabaoth, ce qui ne peut avoir qu'une signification négative ; Épiphane même s'aperçoit de la contradiction (voir Haer. 37, 6, 5-7).

$\mathrm{Si}$ on fait la comparaison avec les sources précédentes, on peut être sûr qu'Épiphane reprend la source du Ps. Tertullien (serpent d'airain, consécration de l'Eucharistie avec le reptile, serpent comme dispensateur de la gnose, création des hommes par les archontes). Il ajoute que les Ophites dérivaient partiellement de Nicolas, ce qui

16. Birger A. Pearson, «Did the Gnostics Curse Jesus?», Journal of Biblical Literature 86 (1967), 301-305. 
n'est pas incompatible avec le cadre donné par le Ps. Tertullien ou avec les Pérates ${ }^{17}$; il intègre aussi le détail rapporté par Irénée, Haer. 1,30,15, sur les intestins humains à forme de reptile. Cette information anatomique pourrait être comparée avec Hippolyte, $E l$. $5,17,11-13$, où l'image du Logos-serpent est devinée dans la forme de l'appareil nerveux. Mais toutes ces données, sauf la création de l'homme, ne s'accordent pas avec la première partie de la notice, où l'évêque de Salamine relate le mythe de Prounikos et ses annexes, d'une façon égale aux Ophites d'Irénée: il s'agit probablement d'un télescopage entre deux sources différentes, sources qui étaient encore bien distinguées chez Irénée, mais situées l'une derrière l'autre. Cette fusion est observable déjà chez le Ps. Tertullien et expliquerait aussi la contradiction sur le rôle du reptile dans l'Eden ${ }^{18}$.

Je propose donc que les deux descriptions données par Irénée correspondent à deux phases différentes du développement de l'ophitisme : une, plus audacieuse et ancienne, axée sur l'exégèse du serpent d'airain et sur l'identification Logos $=$ serpent ; l'autre, postérieure et «docétique », dans les termes de Kaestli, qui aurait progressivement vidé de sa positivité le serpent du Paradis terrestre, pour le ramener à une démonisation typiquement chrétienne et plus traditionnelle. Au premier système appartiendraient l'importance accordée au serpent d'airain, son identification avec le Logos, la célébration de l'Eucharistie avec le reptile, le détail anatomique sur les intestins serpentiformes, le concept de serpent donneur de gnose, une conception positive du péché de Genèse; au deuxième système il faudrait ramener, par contre, le mythe de Prounikos, les fils de Ialdabaoth, la création de l'homme. Dans ce dernier système, on observe qu'Ève crut au serpent envoyé par Ialdabaoth « comme s'il était le fils de Dieu» (Eva autem quasi a Filio Dei hoc audiens, facile credidit) : donc le serpent donneur de gnose, le « discours

17. Voir infra.

18. Cette contradiction pourrait être expliquée en rappelant que le Serpent agit sous l'impulsion de Sophia-Prounikos ; mais, en tout cas, on ne voit pas dans quel but le Démiurge l'aurait envoyé « tromper » Ève. Le problème reste et s'agrandit si on prend en considération l'ensemble des renseignements, qui donnent le portrait, d'un côté, d'un système axé sur le serpent et sur le Logos, de l'autre sur Sophia et sur son intromission dans le reptile. 
de sagesse ", le sophos Logos d'Ève (cf. notice sur les Pérates, $E l .5,16,8)$, expression de la réalité pneumatique dans le premier système, devient ici un être psychique et dérivé du Démiurge, de niveau inférieur. On a l'impression que ces Ophites ne se sont pas seulement ralliés à une exégèse plus conformiste de cet animal, mais qu'ils adressent aussi une polémique voilée à leurs prédécesseurs, coupables d'avoir identifié le Logos avec le serpent. Par contre, les «prédécesseurs » pourraient coïncider avec les Pérates ${ }^{19}$.

Pour ce qui concerne le mythe de Prounikos, il est absent de la notice sur les Pérates, mais il reflète aussi une phase postérieure du développement théologique. En fait, la triade pératique, en accord avec un modèle « binaire » archaïque, oppose le Père et le Logos à la matière (cf. El. 5,12). Prounikos-Sophia, au contraire, procède d'une évolution de la pensée qui ajoute une réflexion sur la troisième Personne de la triade, la Première Femme, véritable essai embryonnaire de pneumatologie ${ }^{20}$.

Philastre s'exprime en des termes qui rappellent Irénée, Haer. 1,30,15: le serpent serait vénéré par les Ophites, car il a donné la sagesse à la femme. Théodoret enfin $($ Haer. 1,14$)$ poursuit l'ouvrage de conflation des sources et identifie les Séthiens avec les Ophites : il donne de nouveau le récit de Prounikos, de la création de l'homme (information coupée partiellement) et de la génération de l'être serpentiforme par le Démiurge, à côté de la consécration de l'Eucharistie grâce au serpent et de l'idée que les intestins ont la forme d'un reptile. Il reprend donc le même récit que le Ps.Tertullien et Épiphane.

On peut en tirer les conclusions provisoires suivantes :

1. Les Pérates sont probablement les premiers Ophites. D'après l'Elenchos, en fait, leur fondateur est Euphrate, homonyme de celui de toute la branche ophite.

19. L'idée que les Pérates pouvaient être identifiés avec les Ophites d'Épiphane avait déjà effleuré Hans Leisegang, La Gnose..., 107.

20. Suggestion orale faite par Josip Montserrat-Torrents; voir surtout Antonio Orbe, La teologia dei secoli II e III: il confronto della Grande Chiesa con lo gnosticismo (trad. it.), Casale Monferrato, Piemme-Rome, Editrice Pontificia, 1995 (éd.originale espagnole Rome, Pontificia Università Gregoriana, 1987), 139-144. 
2. De plus, ils sont les uniques Ophites auxquels on attribue un fondateur quelconque. Même les Naassènes, dont on a cru souvent que les Pérates découlaient ${ }^{21}$, ne partagent pas ce privilège.

3. Ils sont les uniques Ophites qui adorent le serpent véridiquement et qui lui attribuent une importance centrale dans leur conception religieuse ; et cela, sur la base du passage fondamental du serpent d'airain, en développement libre d'une ligne théologique issue de l'école johannique.

4. Selon cette perspective, le serpent est à identifier avec le Logos, descendu sur terre pour le salut des hommes ; chaque apparition du serpent dans l'Ancien Testament l'annonce et le révèle.

5. Pour cela, et selon une ligne de pensée antijuive, on a pu voir dans le serpent du Paradis terrestre une figure positive, le Logos même qui donnait la sagesse aux protoplastes ; le Dieu de l'Ancien Testament, le Démiurge, serait donc apparu méchant et autoritaire. Sur la base de $J n$. 3,14 la transgression de Gn. 3 doit être ainsi considérée comme un moment de révélation du Logos et de rébellion au Démiurge ${ }^{22}$.

6. Pour établir ce lien, les Pérates se sont fondés sur un testimonium qui associait la croix, le bois, le bâton et le serpent : il s'agit d'un recueil d'origine juive, certes, mais qui a dû passer par le christianisme, comme on le voit par l'importance centrale de $J n$. 3,14. Les Pérates étaient des chrétiens ou pouvaient se considérer comme tels.

7. Le Ps.Tertullien, qui nous décrit un groupe axé sur le serpent d'airain, parle vraisemblablement des Pérates. Il nous offre aussi l'information, très cohérente avec cet ensemble, que ces gnostiques utilisaient le serpent pour consacrer l'Eucharistie. Cela est possible seulement si serpent $=$ Logos .

8. Épiphane décrit le même groupe. Pour lui aussi les Ophites consacrent l'Eucharistie avec le serpent et identifient ce dernier avec le Logos.

21. Voir par exemple Robert P. Casey, « Naassenes and Ophites », Journal of Theological Studies 27 (1926), 374-387.

22. Il ne faut pas oublier que les deux épisodes étaient déjà en antithèse dans l'exégèse de Philon : cf. Agric. 94-99 et Leg.all. 3, 78-81. 
9. Le Ps.Tertullien et Épiphane (sans oublier Théodoret) rapportent aussi l'ensemble sur Prounikos et la création de l'homme, qui appartient par contre à un autre système, celui décrit comme ophite par Irénée, Haer. 1, 30,1-14.

10. Personne ne réussira plus, même dans le gnosticisme ophite, à égaler l'audace d'Euphrate : les autres notices montrent qu'une conception docète a progressivement vidé le serpent de son rôle de Logos. Même les hérétiques les plus téméraires n'ont pas été capables d'accepter longtemps l'identification du Christ avec l'image principale de son ennemi direct, le diable.

11. On pourrait donc supposer que les Ophites d'Irénée appartiennent à une phase ultérieure du développement de la branche : toutefois, il ne faut pas oublier que les renseignements sur ces groupes étaient rarement directs, et restent assez vagues.

12. Le développement « docétique » pourrait avoir abouti à une véritable démonisation du serpent, en alignement avec la tradition chrétienne ; on en a un souvenir dans le récit des Ophites d'Irénée et, probablement, dans la contradiction dans laquelle tombe la notice d'Épiphane.

Le parcours envisagé par Kaestli se révélerait donc trop simple : l'ophitisme pourrait être issu, en premier lieu, depuis un courant d'ascendance johannique ; mais, cette identification du serpent avec le Logos aurait été ensuite mitigée, par influence d'autres conceptions chrétiennes plus traditionnelles, qui voyaient dans cet animal simplement le symbole du mal.

Dans ce cadre, J. Charlesworth a probablement raison, lorsqu'il propose, dans son interprétation de Jn. 3,14, l'existence d'une ophidian christology : une « christologie ophite » fondée sur l'identification de Jésus avec le serpent d'airain, issue de l'école johannique, mais qui atteignit son paroxysme parmi les Ophites ${ }^{23}$. Toutefois, Euphrate est resté seul avec son audace téméraire, ce qui laisse

23. James H. Charlesworth a exposé sa conception de la ophidian christology lors d'une très récente journée d'études bibliques à l'Université de Genève (Ophidian Symbology and Biblical Theology, le 16 avril 2005) et dans un ouvrage encyclopédique, The Good and Evil Serpent, Anchor Bible, en cours d'impression et dont la parution est prévue pour le mois de juin 2008. 
supposer que l'ophitisme est né avec les Pérates, autour du milieu du $\mathrm{II}^{\mathrm{e}}$ siècle ou même auparavant.

Je n'ai pas mentionné jusqu'ici les Naassènes. Une simple lecture de leur notice laisse entrevoir leur parenté étroite avec les Pérates. Je suppose, sur la base des arguments exposés plus haut, qu'ils dérivent de l'autre groupe : et j'ajoute un autre détail. Il est vrai que leur nom provient directement du serpent : selon le rédacteur de l'Élenchos, il dérive de l'hébreu nahas, «serpent » (cf. 5, 6,3-4); mais le jeu de mots avec le grec naos, «temple », leur permettrait d'affirmer que tous les temples et les religions du monde existent grâce au serpent et lui sont dédiés, car il n'y a pas de religion sans temples ni serpents (cf. 5, 9,12) ${ }^{24}$. Le jeu de mots entre nahas et naos semble impliquer la procédure juive du ma'al, une sorte de paronomase, déjà observée pour les Pérates ${ }^{25}$. Cela, comme d'ailleurs toute la notice, confirme la stricte parenté entre Naassènes et milieu juif. Malgré les déclarations d'El. 5, 6, 3, selon lesquelles les Naassènes auraient été les premiers à vénérer les serpents, je préférerais donner à cette affirmation une valeur relative. En fait, je crois que

24. Mark J. Edwards, «The Naming of the Naassenes: Hippolytus, Refutatio V.6-10 as Hieros Logos », Zeitschrift für Papyrologie und Epygraphik 112 (1996), 74-80, refuse cette étymologie et propose de voir dans le nom Naassènes le résultat de naos, «temple », et nous, « cerveau, esprit » : mais il n'est pas en mesure de prouver ce passage au niveau phonétique. De plus, il suppose gratuitement que cette étymologie est le produit de la hargne des hérésiologues, car le serpent serait un perverse object of worship (cit. 75) : c'est ignorer ainsi l'importance universelle du reptile dans les religions anciennes. Cette perspective s'appuie en outre sur la reconstruction du texte jadis avancée par Reitzenstein et sur l'idée que les Naassènes découleraient d'un prégnosticisme : pour cela, les insertions chrétiennes devraient être considérées comme postérieures. Un simple regard à la notice suffit pour confirmer les liens étroits existant entre Naassènes et judéo-chrétiens, sans oublier que, l'Elenchos le dit clairement, leur nom vient de nahas.

25. Cette procédure souligne l'identité phonétique et la divergence sémantique de deux mots, pour établir des significations nouvelles : Annarita Magri, «L'esegesi della setta ofitica dei Perati. Analisi di Hipp.Haer. V,16 », Apocrypha 14 (2003), 193-223, surtout 218 ; Lou H. Silberman, « A Note on 4Q Florilegium », Journal of Biblical Literature 78 (1959), 158-9 et Wilhelm Bacher, Die Exegetische Terminologie der jüdischen Traditionsliteratur, Leipzig, J. C. Hinrich'schen Buchhandlung, 1905, 111-112. 
cette focalisation sur l'animal a été progressive : les Naassènes pourraient avoir été les premiers à assumer une dénomination explicite tirée du serpent et à apparaître ainsi comme ses adorateurs officiels, mais l'idée pourrait être née dans un autre groupe. En tout cas, leur notice est trop longue et détaillée pour être analysée ici, raison pour laquelle je me propose d'aborder le problème ailleurs.

Toutefois, l'importance accordée au serpent par les Pérates et les Ophites ne peut pas être expliquée seulement sur la base des données judéo-chrétiennes : dans cet horizon, une vision fortement négative du reptile prévalait depuis longtemps. Il faut donc chercher dans l'environnement où ces groupes vivaient pour comprendre si la culture et la religion contemporaines proposaient quelque chose d'analogue. Or, il se trouve que justement pendant le $\mathrm{II}^{\mathrm{e}}$ siècle le serpent était devenu une figure extrêmement populaire, presque « à la mode ». Cela nous met dans la condition de pouvoir expliquer l'origine des Pérates et de l'ophitisme.

\section{LE SERPENT « À LA MODE » AU II ${ }^{\mathrm{E}}$ SIÈCLE}

Si on passe en revue la documentation archéologique contemporaine des Pérates, on est étonné par la fréquence avec laquelle le motif du serpent apparait sur les objets les plus divers : décorations architecturales, joyaux, monnaies, objets de culte. J'en donnerai seulement quelques échantillons.

P. Romanelli a identifié sur un chapiteau ionique une décoration très rare qui consistait en deux serpents entrelacés : il la date entre la deuxième moitié du $\mathrm{II}^{\mathrm{e}}$ siècle et le début du $\mathrm{III}^{\mathrm{e}} 26$.

D. Alicu a constaté l'omniprésence de la vaisselle sacrée décorée de serpents en Dacie, région qui fut colonisée, on le sait, pendant le II $^{\mathrm{e}}$ siècle.

26. Pietro Romenelli, «Un Modiglione di Leptis Magna con decorazione di nodo di serpenti », American Journal of Archaeology 66 (1962), 313-315. 
La présence de ces vases dans des milieux si divers, dans des temples différents, dédiés à des divinités qui ne se trouvent pas en rapport direct avec Mithra et Sabasius, indique l'utilisation de ces vases sur un large territoire, à partir de la seconde moitié du $\mathrm{II}^{\mathrm{e}}$ siècle $^{27}$.

Il ne s'agit pas d'un motif artistique spécialement lié à une divinité particulière, mais d'un thème répandu parmi de nombreux cultes très divers, si répandu que J. Collins-Clinton a même forgé un terme $a d$ hoc, « snakeware ${ }^{28}$. On en voit les reflets, à la même époque, aussi dans la fabrication de bijoux ${ }^{29}$.

La popularité du serpent dans la vaisselle cultuelle nous conduit à la véritable raison de sa renommée à cette époque : sa présence extrêmement fréquente dans les cultes, surtout à mystères, d'où la facies ainsi caractéristique de cette période.

Le serpent est un animal avant tout chthonien, lié à la terre et aux rites archaïques des déesses mères ; il apparaît dans la phase la plus ancienne du développement des religions de la Méditerranée, au Moyen Orient et dans l'Égée. Son regard aigu, perçant, a donné une réputation considérable à ses capacités visuelles et, en conséquence, a forgé le mythe de sa ruse ; il est donc devenu l'animal mantique par excellence et un symbole prégnant de sagesse. Pendant le $\mathrm{II}^{\mathrm{e}}$ siècle, par contre, c'est une autre de ses qualités qui excitait l'intérêt : la mue, donc sa possibilité de renaître chaque année et de changer sa peau après la phase de léthargie en hiver. Cette caractéristique a fait du reptile l'animal sacré de maints cultes à mystères, qui promettaient, justement au II $^{\mathrm{e}}$ siècle, l'immortalité de l'âme et le salut dans l'Au-delà. C'est l'époque d'une véritable « révolution religieuse », qui vit grandir les préoccupations spirituelles : dans ce cadre, l'animal considéré depuis longtemps comme porteur de vie,

27. Dorin Alicu, «Vases décorés de serpents, découverts à Sarmizegetusa », Latomus 39, (1980), 717-725, cit. 725.

28. Voir Jacquelyn Collins-Clinton, A Late Antique Shrine of Liber Pater at Cosa (Études préliminaires aux religions orientales dans l'Empire Romain 64), Leyde, Brill, 1977, 24.

29. Hilary E. M. Cool, « The Significance of Snake Jewellery Hoards », Britannia 31 (2000), 29-40. 
jeunesse et renaissance, devint un des symboles religieux les plus puissants ${ }^{30}$.

\section{LE SERPENT D’ASCLÉPIOS}

Tracer une liste des cultes qui ont utilisé le symbole du serpent demanderait une véritable encyclopédie. La stratification de ses significations religieuses le rend présent, soit comme animal chthonien, soit comme symbole mantique, ou encore de renaissance et d'immortalité, dans presque tous les rituels des divinités anciennes. Mais l'un d'eux était probablement plus populaire que les autres : cela aiderait ainsi à expliquer l'identification par les Pérates du Logos et du reptile. Il s'agit du culte d'Asclépios, le dieu guérisseur le plus connu de la culture gréco-romaine, qui atteint le sommet de sa popularité précisément au $\mathrm{II}^{\mathrm{e}}$ siècle.

Figure originaire de la Thessalie, reconnue comme divinité entre le $\mathrm{VI}^{\mathrm{e}}$ et le $\mathrm{V}^{\mathrm{e}}$ siècle avant Christ, Asclépios devint le dieu guérisseur par excellence du Panthéon gréco-romain ${ }^{31}$. Il passait pour le fils

30. La bibliographie sur les valeurs symboliques attribuées à cet animal, soit dans les écritures juives et chrétiennes, soit dans la littérature païenne, est très vaste : je me borne ici à indiquer Edgar Küster, Die Schlange in der griechischen Kunst und Religion (Religiongeschichtliche Versuche und Vorarbeiten 13), Giessen, Töpelmann, 1913 ; Maria Lucia Sancassano, Il Serpente e le sue immagini. Il motivo del serpente nella poesia greca dall'Iliade all'Orestea (Biblioteca di Athenaeum 36), Côme, New Press, 1997, et surtout James H. Charlesworth, The Good and Evil Serpent, en cours d'impression.

31. Pour un recueil des sources, Emma Jeannette Edelstein, Ludwig Edelstein, Asclepius. A Collection and Interpretation of the Testimonies, Baltimore, The Johns Hopkins Press, 1945 ; aussi Gregory Vlastos, « Religion and Medicine in The Cult of Asclepios: A Review Article », Review of Religion 13 (1948), 269-290, qui corrige Edelstein. Pour le dieu et sa vénération, Eduard Thrämer, «Asklepios », dans Paulys Realencyclopädie der classischen Altertumswissenschaft (neue Bearbeitung), vol. 2, 2, 1896, 1642-1697 ; Ulrich von Wilamowitz-Moellendorff, Der Glauben der Hellenen, vol. I, Darmstadt, Wissenschaftliche Buchgesellschaft, 1994, réimpression de 1955², 220-230, qui considère le serpent comme un trait très archaïque. L'étymologie du nom du dieu serait pélasgique selon Vladimir I. Georgiev, « Aiskalapios-Asklepios », Acta Antiqua Academiae Scientiarum Hungaricae 25 (1977), 317-319; on a proposé par ailleurs une dérivation de askalabos, « lézard », en Eduard Thrämer, 
d'Apollon et de la princesse mortelle Coronis : initié par le centaure Chiron à l'art médical et devenu expert au point d'être capable de ressusciter les morts, il fut pour cela foudroyé par Zeus ${ }^{32}$.

Comme on l'a dit, la popularité d'Asclépios a atteint son acmé au II ${ }^{\mathrm{e}}$ siècle, lorsqu'il fut même identifié avec Zeus ${ }^{33}$ : à cette époque,

« Asklepios », 1643. Les savants sont partagés à propos de l'origine de cette divinité : il s'agit ou bien d'un héros divinisé ou d'une divinité archaïque chtonienne. Pour l'histoire du mythe, voir Gregory Vlastos, «Religion and Medicine...»; René Josef Ruettimann, Asclepius and Jesus: The Form, Character and Status of The Asclepius Cult in The Second-Century CE and its Influence on Early Christianity, Dissertation discussed at the Harvard University, 1987, Ann Arbor, Michigan, University Microfilm International, 1989, 12-21 ; Alice Walton, Asklepios. The Cult of the Greek God of Medicine, Chicago, Ares Publishers Inc., 1979, 1-35 (avec une liste des sources aux pages 85-95); Jan Schouten, Serpent of Asklepios. Symbol of Medicine (trad. anglaise de l'original néerlandais), Amsterdam-Londre-New York, Elsevier Publishing Co., 1967, 7-22 ; Vincenzo Cilento, «Asclepio », La Parola del Passato 21 (1966), 449-459 ; Kàroly Kerenyi, Asklepios. Archetypal Image of the Physician's Existence (trad. anglaise) (Bollingen Series 65.3), New York, Bollingen Foundation-Pantheon Books, 1959 (éd. originale allemande Bâle, Ciba Ldt., 1947), 87-101. Pour une réflexion sur les origines de ce mythe, Giannina Solimano, Asclepio. Le aree del mito (Pubblicazioni dell'Istituto di Filologia Classica e Medievale 46), Gêne, Facoltà di Lettere, Istituto di Filologia Classica e Medievale, 1976 ; Christa Benedum, « Asklepiosmythos und archäologischer Befund », Medizinhistorisches Journal 22 (1987), 48-61 et Christa Benedum, «Asklepios-Der homerische Artz und der Gott von Epidaurus », Rheinisches Museum für Philologie 133 (1990), 210-226. Selon Giannina Solimano, le status divin ou héroïque dépend surtout de la phase d'évolution religieuse d'une croyance: Asclépios aurait donc été, à l'origine, un dieu-serpent de la Thessalie, anthropomorphisé par la suite. Cet avis est partagé par Peter Robert Franke, «Asklepios - Aesculapius auf antiken Münzen », Medizinisches Monatsspiegel MERCK 14 (1969), 60-67, surtout 61-62. Christa Benedum situe, par contre, la divinisation d'un héros homérique, toujours thessalien, pas avant le $\mathrm{VI}^{\mathrm{e}}$ siècle : par la suite, le développement du culte d'Épidaure aurait signifié une nouvelle organisation de cette vénération à partir du ve-IVe siècle.

32. Voici une petite liste des sources principales sur le mythe : Homère, Il. 4,192-219; Hymne 16,1-5 ; Hymne orphique 67, Pindare, Pyth. 3,1-58; Platon, Rép. 407-8, Diodore 4, 71 ; ib. 5, 74 ; Paus. 2, 26, 3-10 et 8, 25, 11 ; Apollodore Bibl. 3, 10, 3 ; Vergile Én. 7, 760-83 ; Ovide, Mét. 2, 542-48; ib. 15, 531-46 et 622-744 ; Fas. 6, 743-54 ; Hygin, Fab. 49 et Astr.14, 5, etc.

33. René Josef Ruettimann, Asclepius and Jesus... ; Ulrich von WilamowitzMoellendorff, Der Glauben der Hellenen ..., vol. I, 497-500 ; Howard Clark Kee, "Self-Definition in the Asclepius Cult », dans Ben F. Meyer and Ed P. Sanders éd., Self-Definition in the Graeco-Roman World, vol. III, Londre, SCM Press 
il comptait, autour de la Méditerranée, plus de 500 sanctuaires ${ }^{34}$. Son temple principal se trouvait à Épidaure : c'était un ensemble architectonique restauré par Hadrien ${ }^{35}$. Dans le naos, on pouvait admirer une statue du dieu, appuyé sur son bâton autour duquel un serpent était enroulé ${ }^{36}$. Le dieu était très vénéré aussi en Asie Mineure : le centre le plus fameux était Pergame, restauré à l'époque d'Hadrien ${ }^{37}$; là aussi, il existait une sculpture du dieu, réalisée par Phyromachos, avec le bâton à serpent ${ }^{38}$, motif reproduit sur les

Ltd, 1982, 118-36. Au II ${ }^{\mathrm{e}}$ siècle remontent, par exemple, les inscriptions étudiées par Kent J. Rigsby, « A Roman Epigram for Asclepius », Zeitschrift für Papyrologie und Epygraphik 134 (2001), 107-108 et par Lothar Schwinden, «Die Weihinschrift für Asclepius CIL XIII 3636 aus Trier », Trierer Zeitschrift 57 (1994), 133-145 (période de l'épidémie de peste de 165-80).

34. Eduard Thrämer, « Asklepios », 1662-77, où on nomme 186 sanctuaires, et la liste de Alice Walton, Asklepios..., 95-121 ; Alessandra Semeria, « Per un censimento degli Asklepieia della Grecia continentale e delle isole », Annali della Scuola Normale di Pisa, III serie 16 (1986), 931-958 contient les sources concernant seulement 59 sanctuaires de la Grèce continentale et insulaire.

35. René Josef Ruettimann, Asclepius and Jesus..., 44 et 55 ; sur le temple d'Épidaure, 21-27, ib. 46-54 et Kàroly Kerenyi, Asklepios..., 18-46.

36. Pausanias 2,27,2: la statue, chryséléphantine, avait été sculptée par Thrasymède autour de 370 a.c. ; Peter Robert Franke, « Asklepios - Aesculapius auf antiken Münzen... », 61-62, qui en offre l'image reproduite sur les monnaies de la ville.

37. René Josef Ruettimann, Asclepius and Jesus..., 32 ; sources sur le sanctuaire : 4-5 et 98-101; l'ouvrage de Ruettimann est focalisé sur le $\mathrm{II}^{\mathrm{e}}$ siècle et l'Asie Mineure, où la présence des chrétiens s'affirma très tôt. Un nouveau temple fut bâti grâce au soutien de L. Cuspius Pattumeius Rufinus en 150 sur le modèle du Panthéon : voir Hans-Joachim Schalles, «Pergamon », dans Jane Turner éd., The Dictionary of Art, vol. 24, Londre, Macmillan Publishers Limited, 1996, 410-416, surtout 413 ; Adolf Hoffmann, « Zum Baumplan des Zeus-Asklepios-Tempels im Asklepieion von Pergamon », dans Deutsches Archäologisches Institut, Bauplanung und Bautheorie der Antike (Diskussionen zur archäologischen Bauforschung 4), Drückerei Hellmich KG, 95-103. Le grand nombre d'inscriptions témoigne aussi de la renommée internationale de ce lieu depuis Hadrien.

38. Voir René Josef Ruettimann, Asclepius and Jesus..., 30. Sur la célèbre statue de Phyromachos, voir Bernard Andreae, «Der Asklepios des Phyromachos », dans Bernard Andreae et alii éd., Phyromachos Probleme. Mit einem Anhang zur Datierung des grossen Altares von Pergamôn (Mitteilungen des Deutschen Archaeologischen Instituts Römische Abteilung 31), Mayence, Verlag P.von Zabern, 1990, 45-100 (elle remontait aux années 167-156 a.c.). 
monnaies de la ville asiatique ${ }^{39}$. Les autres sanctuaires importants étaient Cos, restauré aussi par les Antonins ${ }^{40}$, et Rome, pareillement très fréquenté, d'après les inscriptions et les monnaies, pendant la même période ${ }^{41}$ : Christa Benedum lie cet intérêt grandissant au fléau qui ravagea l'Empire sous Marc-Aurèle, entre 165 et $180^{42}$; en outre, depuis Hadrien, le dieu était le protecteur officiel de la santé de l'empereur ${ }^{43}$. Le culte s'adressait avant tout aux malades et impliquait la pratique de l'incubation et l'oniromancie ${ }^{44}$; il pouvait y

39. René Josef Ruettimann, Asclepius and Jesus..., 72-73 et 89.

40. Francesco Sirano, « Considerazioni sull'Asclepio « Tipo Nea Paphos ». Ipotesi su di un gruppo di sculture di età imperiale », Archeologia Classica 46 (1994), 199-232, surtout 226-228.

41. René Josef Ruettimann, Asclepius and Jesus..., 19-20 et 57-64 ; Chiara De Filippis Cappai, «Il culto di Asclepio da Epidauro a Rome : medicina del tempio e medicina scientifica », Civiltà classica e cristiana 12 (1991), 271-84 ; Christa Benedum, «Betrachtungen zu Asklepios und dem Aesculapius der Römer », Würzburger Jahrbücher für die Altertumswissenschaft, Neue Folge 25 (2001), 187-207. Pour les sources littéraires principales, cf. Ovide, Mét. 15, 622-744, Live, 10, 47, 7, Valère Maxime, 1,8,2 et Augustin, Cité de Dieu, $3,17, i b .10,16$. Franke reproduit le médaillon commandé par Antonin le Pieux en mémoire de l'arrivée du serpent sacré sur l'île Tibérine en «Asklepios - Aesculapius auf antiken Münzen... », 65-66.

42. Christa Benedum, «Betrachtungen zu Asklepios...», surtout 199 : selon elle, les monnaies avec les images du dieu pourraient permettre de reconstruire le parcours de l'épidémie.

43. Voir Chiara De Filippis Cappai, «Il culto di Asclepio... », 279-80 ; Francesco Sirano, « Considerazioni... », en particulier 227.

44. Pour la pratique cultuelle et médicale, voir Rudolf Herzog, «Die Wunderheilungen von Epidauros. Ein Beitrag zur Geschichte der Medizin und der Religion », Philologus Suppl. 22, 3, 1-164 (Leipzig, Dieterich, 1931), encore très valable et qui introduit une différence entre la pratique d'Épidaure, plus encline au merveilleux, et celle de Cos, de tendance plutôt scientifique; Fritz Graf, « Heiligtum und Ritual. Das Beispiel der griechisch-römischen Asklepieia », dans Albert Schachter éd., Le sanctuaire grec (Entretiens sur l'Antiquité Classique 37), Genève, Fondation Hardt, 1992, 159-203 ; Alice Walton, Asklepios.., 57-67 et 76-82 ; Jan Schouten, Serpent of Asklepios..., 49-55 ; Gregory Vlastos, «Religion and Medicine... », 286-288; et surtout Guido Guidorizzi, «Sogno, malattia, guarigione : da Asclepio a Ippocrate », dans Istituto di Filologia Classica, Graeco-Latina mediolanensia (Quaderni di Acme 5), Università degli Studi di Milan-Facoltà di Lettere e Filosofia, Milan, CisalpinoGoliardica, 1985, 59-74, retravaillé dans Guido Guidorizzi, « Sogno, diagnosi, 
avoir un rapport assez étroit avec la médecine ${ }^{45}$. Le culte d'Asclépios résista jusqu' au $\mathrm{V}^{\mathrm{e}}$ siècle ${ }^{46}$.

Avant de poursuivre, $\mathrm{j}$ 'aimerais attirer l'attention sur un aspect important de cette dévotion, surtout dans l'Asie Mineure du II siècle : le syncrétisme avec d'autres dieux, en premier lieu Zeus. Kranz a démontré que des traits syncrétistes entre les deux divinités existaient déjà à la fin de l'époque classique, mais le phénomène toucha à son sommet dans l'ambiance asiate, en particulier Pergame, au II ${ }^{\mathrm{e}}$ siècle $^{47}$. Cette «théocrasie », comme la définit Bonner, permit à Asclépios d'absorber les qualités de Zeus et Dionysos et s'inscrit dans la

guarigione : da Asclepio a Ippocrate », dans Guido Guidorizzi éd., Il sogno in Grecia, Rome-Bari, Laterza, 1988, 87-102 ; Luciano Bonuzzi, « Angoscia e malattia nei santuari di Asclepio e alle origini del pellegrinaggio cristiano », dans Antje Krug éd., From Epidaurus to Salerno. Symposium held at the European University Centre for Cultural Heritage, Ravello, April 1990, Conseil de l'Europe-Division de la Coopération scientifique, PACT 34 (1992), Rixensart, PACT Belgique, 1992, 51-59. Pour un essai d'interprétation moderne de ces pratiques, voir Rudolf Herzog, « Die Wunderheilungen von Epidauros... »; Emma Jeannette Edelstein-Ludwig Edelstein, « Asclepio: la medicina del tempio », dans Guido Guidorizzi éd., Il sogno in Grecia..., 67-86, synthèse tirée de leur ouvrage majeur; Vincenzo Longo, «I segreti del tempio di Asclepio in Epidauro: tradizione religiosa e tradizione laica », Atti dell'Accademia Ligure di Scienze e Lettere 48 (1992), 437-446, dont les conclusions ne sont pas convaincantes.

45. La connexion entre culte et médecine reste obscure pour les modernes : elle variait selon la tradition des sanctuaires (pour cette idée, voir Rudolf Herzog, «Die Wunderheilungen von Epidauros... »); mais il faut rappeler que les anciens n'y voyaient pas la contradiction aperçue aujourd'hui, surtout parce que les miracles étaient censés appartenir au cours naturel des choses : René Josef Ruettimann, Asclepius and Jesus..., 18-19, ib. 85 et 115-121 et la bibliographie citée dans la note précédente. Kee, par contre, insiste sur le côté irrationnel de ces dévotions, un trait vraisemblablement adapté au $\mathrm{II}^{\mathrm{e}}$ siècle : Howard Clark Kee, « Self-Definition in the Asclepius Cult... », 129 et 136.

46. René Josef Ruettimann, Asclepius and Jesus..., passim; Christa Benedum, «Betrachtungen zu Asklepios und dem Aesculapius der Römer... », 202-204.

47. Peter Kranz, «Die Asklepiosstatue im Schlosspark von Klein-Glienicke. Ein neuer Typus und sein kulturgeschichtlicher Hintergrund », Jahrbuch des Deutschen Archäologischen Instituts 104 (1989), 107-155. Il faut remarquer que, dans ce processus, le bâton du dieu est souvent assimilé à un objet appartenant à une autre divinité, comme le sceptre. En général, voir Rudolf Herzog, " Asklepios », dans Theodor Klauser éd., Reallexikon für Antike und Christentum, vol. I, Stuttgart, Hiersemann Verlags-GMBH, 1950, 795-799, surtout 796. Christa 
religiosité hénothéiste de l'époque ${ }^{48}$. Cela est très important pour comprendre comment le dieu guérisseur put rejoindre le niveau de la divinité suprême.

À la même période on assista à un autre changement : le dieu, à côté de son rôle de guérisseur, devint pour ses fidèles un guide spirituel dans toutes les situations de la vie ${ }^{49}$.

It can be shown that in the cult of the Divine Healer there was developed to a high point a faith in a providence which did not disdain to concern itself with the intimate life of the individual - not only his body ailments but also his spiritual well-being ${ }^{50}$.

Asclépios était donc: «a personal Savior with far-reaching attributes, and the object of the most devout adoration ${ }^{51} »$. Cela entraîna une dure réaction de la part des chrétiens.

Parmi les divinités païennes, Asclépios était sûrement la plus proche de la figure de Jésus. Déjà Dölger, il y a plusieurs décennies, a étudié ce conflit : les sources de la polémique chrétienne contre ce dieu remontent souvent aux discussions des écoles philosophiques classiques $^{52}$. Ruettimann a énuméré les traits d'après lesquels le fils d'Apollon ressemble à Jésus :

1. Les deux sont des dieux guérisseurs et sauveurs.

2. Ils sont envoyés par la divinité suprême sur la terre, pour accomplir leur mission.

3. Ils sont engendrés par un dieu et une femme mortelle.

Benedum ajoute que ce syncrétisme pourrait avoir évolué depuis l'association, vivante à Athènes, entre Asclépios, Zeus Ktesios, Meilichios et Philios, trois épiclèses du dieu suprême associées à l'attribut du serpent et à la protection de l'individu : Christa Benedum, «Asklepiosmythos... ».

48. Campbell Bonner, « Some Phases of Religious Feeling in Later Paganism », Harvard Theological Review 30 (1937), 119-40 ; Howard Clark Kee, «Self-Definition in the Asclepius Cult... », 134.

49. Voir Howard Clark Kee, «Self-Definition in the Asclepius Cult... », et Alice Walton, Asklepios..., 66-67. Aristide en est un cas emblématique.

50. Campbell Bonner, « Some Phases of Religious Feeling... », cit. 123.

51. Ibid., cit. 124.

52. Franz Dölger, « Der Heiland », Antike und Christentum 6 (1940-1950), 241-272. Dölger, en revanche, ne reconnaît pas le rôle de sauveur spirituel auquel Asclépios parvint au $\mathrm{II}^{\mathrm{e}}$ siècle : 253. 
4. Leur mission consiste dans une aide philanthropique aux êtres humains.

5. Ils sont capables de guérir.

6. Ils accomplissent même des résurrections de morts.

7. Leur abnégation les pousse à s'occuper surtout des plus délaissés.

8. Ils sont caractérisés par un sens élevé de la moralité.

9. Ils sont tués à cause de leur mission (Asclépios, parce qu'il avait troublé l'ordre naturel en ressuscitant des défunts ; Jésus, à cause de la résurrection de Lazare).

10. Après leur mort, ils apparaissent à leurs disciples ${ }^{53}$.

11. Puis ils sont élevés au ciel.

12. Ils sont donc considérés comme des divinités.

13. Ils laissent des disciples pour poursuivre leur mission.

14. Ces derniers continuent à utiliser les mêmes pouvoirs que leurs maîtres et à accomplir des guérisons ${ }^{54}$.

J'ajouterai aussi que l'éthique était au cœur des préoccupations des adeptes des deux religions et que plusieurs sanctuaires dédiés à Asclépios furent ensuite transformés en lieux de culte chrétien ${ }^{55}$.

53. Les apparitions d'Asclépios sont celles rappelées dans les documents épigraphiques d'Épidaure du IVe siècle a.c.

54. René Josef Ruettimann, Asclepius and Jesus..., 194-208. La ressemblance était telle que les humanistes, pour éviter des noms sémitiques dans leurs ouvrages, appelaient Jésus « Asclépios » : voir Eduard Norden, La prosa d'arte antica dal VI secolo a.C. all'età della Rinascenza (trad. it.), Rome, Salerno ed., 1986² (éd. originale allemande Leipzig, 1898), 779, note 26.

55. Jan Den Boeft a nié récemment le caractère exclusif de l'opposition entre Jésus et Asclépios, mais d'une façon peu convaincante : Jan Den Boeft, «Christ and Asklepios », Euphrosyne NS 25 (1997), 337-342. Il est clair que tous les dieux païens étaient à considérer, par les chrétiens, comme des ennemis, mais Asclépios dut avoir un rôle particulier dans cette opposition. La comparaison entre Jésus et Asclépios est admise en revanche par d'autres savants, comme Van Staden, qui donne ces analogies: naissance d'un dieu et d'une femme mortelle, risque de mourir après la naissance, philanthropie, mort tragique, épiphanie post mortem, ascension au ciel et divinisation. Le savant, toutefois, ajoute des arguments discutables, comme une réduction excessive de la figure de Jésus à un mythe et la psychologisation d'Asclépios. De plus, il soutient que l'histoire de Jésus dépendrait de celle d'Asclépios (cf. 102), ce qui méconnaît 
Jésus et Asclépios, dieux bienfaiteurs et sauveurs de l'humanité, salués tous les deux comme soteres ${ }^{56}$, pouvaient donc être considérés presque équivalents. À propos de ce titre, la recherche la plus approfondie demeure celle de Dölger : il rappelle que Justin connaissait la signification du prénom « Jésus »= Sauveur (I Apol. I, 33,7) et suppose que cet attribut se répandit dans sa forme absolue parmi chrétiens, orthodoxes et hérétiques, autour du milieu du $\mathrm{II}^{\mathrm{e}}$ siècle ${ }^{57}$. En fait, la médecine était alors comprise parmi les tâches de la religion et le salut de l'âme impliquait celui du corps ${ }^{58}$.

J'aimerais souligner ici l'importance du milieu asiate à cet égard. Pergame, deuxième patrie d'Asclépios, était aussi un centre de développement de l'Église, au moins depuis la fin du I ${ }^{\mathrm{er}}$ siècle : nous pouvons nous en rendre compte grâce à l'Apocalypse et par Eusèbe, qui signale plusieurs martyrs provenant de cette ville (cf. $H E$ 5,1, 17, 37-52 $)^{59}$. De plus, Ruettimann suppose qu'Ignace d'Antioche, le premier à appeler Jésus iatros en Eph. 7,1-2, y conteste des fidèles du dieu grec. Éphèse était en fait un centre important du culte

les racines juives du christianisme. En fait, les fouilles effectuées à Jérusalem sur le lieu supposé de la Piscine Probatique (cf. Jn. 5) ont révélé l'existence d'un probable sanctuaire de guérison sémitique, dont la place fut prise, après 70 (phase d'Aelia Capitolina) par un temple d'Asclépios-Sérapis: voir Antoine Duprez, Jésus et les dieux guérisseurs. À propos de Jean, V (Cahiers de la Revue Biblique 12), Paris, Gabalda, 1970 ; P. J. Van Staden, « Jesus and Asklepios », Ekklesiastikos Pharos 80 (1998), 84-111.

56. Pour ce titre, voir René Josef Ruettimann, Asclepius and Jesus..., 82 et 146-51. D'après Georgiev, il est le plus fréquemment utilisé pour la divinité d'Épidaure: Vladimir I. Georgiev, « Aiskalapios-Asklepios », 318 ; cela est confirmé par le specimen de Alice Walton, Asklepios..., 84 ; voir aussi Ulrich von Wilamowitz-Moellendorff, Der Glauben der Hellenen ..., vol. II, 464.

57. Franz Dölger, « Der Heiland ». Ce titre semble plus diffusé parmi les gnostiques, Naassènes, Pérates (1 occurrence), dans l'Épître à Flora de Ptolémée (11 fois), dans le commentaire de Jean d'Héracléon (23 fois) ; aussi Valentin en Irénée, Haer. 1,1, 3 et 9 et Clément d'Alexandrie, Stromates 3,13,92,1, à propos de Jules Cassien. Il pourrait donc s'agir d'une innovation gnostique, ou d'un usage plus diffus dans ces ambiances. Du côté orthodoxe on peut mentionner Quadratus (cf. Eusèbe, $H E$ 4, 3,1-2), Ignace, Phil. 9, 2 (mais l'attribut manque dans la version brève), Justin, Méliton, Tatien, etc. L'Asie semble donc la mieux représentée.

58. Voir René Josef Ruettimann, Asclepius and Jesus..., 5.

59. Voir Id., ibid., 91. 
d'Asclépios, comme on le voit par les monnaies et par l'existence d'un concours annuel pour les médecins ${ }^{60}$ : la définition de Jésus iatros aurait donc une allure polémique ${ }^{61}$. Mais, même sans cette référence, de nombreux autres témoignages confirment une opposition chrétienne aiguë 62 : cette opposition laisse comprendre quelle sorte de danger Asclépios représentait pour le christianisme, un danger syncrétiste réel.

Enfin, il est possible de repérer une certaine continuité iconographique entre Asclépios et des figurations de Jésus datables entre la fin du III et le début du IV siècle : il s'agirait surtout du portrait du Christ barbu, représenté à la façon d'un philosophe ${ }^{63}$. D'après le spécialiste de l'iconographie J. Schouten, le rapport entre les deux types demeure une possibilité indémontrable : en fait, à cette époque la barbe était courante, en tant que synonyme de pouvoir ${ }^{64}$. Toutefois, des recherches plus récentes ont apporté des éléments plus sûrs, surtout à la lumière de l'équivalence formelle entre le salut chrétien et celui donné par Asclépios et de l'importance accordée à la guérison pendant l'époque impériale ${ }^{65}$.

Le symbole iconographique le plus commun du dieu est le bâton avec un serpent enroulé autour : le bâton appartenait en fait aux médecins ambulants, mais il possédait sûrement des significations

60. Voir Dieter Knibbe, «Ephesos- nicht nur die Stadt der Artemis », dans Sencer Sahin, Elmar Schwertheim éd., Studien zur Religion und Kultur Kleinasiens : Festschrift für Friedrich Karl Dörner zum 65. Geburtstag am 28. Februar 1976 (Études préliminaires aux religions orientales dans l'Empire Romain 66, 2), Leyde, E. J. Brill, 1978, 489-503, surtout 494.

61. Voir René Josef Ruettimann, Asclepius and Jesus..., 164-72.

62. Voir par exemple Tertullien, Adversus Nationes. 2,14 et Lactance, Divinae Institutiones 1,19, 3-4 ; ib. 2, 6,11 ; ib. 4, 27, 12.

63. René Josef Ruettimann, Asclepius and Jesus..., 180.

64. Jan Schouten, Serpent of Asklepios..., 70 : le type du Christ barbu, avec les cheveux partagés au milieu, a survécu jusqu'à aujourd'hui.

65. Voir Erich Dinkler, Christus und Asklepios. Zum Christustypus der polychromen Platten im Museo Nazionale Romano (Sitzungberichte der Heidelberger Akademie der Wissenschaften, Philosophisch-historische Klasse 1980/2), Heidelberg, C.Winter-Universitätsverlag, 1980 ; Christa Benedum, Betrachtungen zu Asklepios..., 203. Dinkler cite aussi le cas d'une tête du dieu grec, qui fut réutilisée comme portrait du Christ dans une église de Gerasa au début du v viècle et aujourd'hui conservée au Musée National d'Amman : 33. 
plus complexes ${ }^{66}$. Thrämer y voit une valeur religieuse ${ }^{67}$; selon G. Solimano, il pourrait correspondre à un sceptre, donc être un emblème de pouvoir ${ }^{68}$. Kerenyi observe :

(The staff) does not differ essentially from a Greek King's scepter, which is nothing other than a staff that was handed down from generation to generation in noble- in Homeric times royal-families. The snake twined round the staff of Asklepios is a second attribute whose symbolism, pointing to the origin of the family in question (the Asclepiads), coincides with that of the staff itself ${ }^{69}$.

Et C. De Filippis Cappai ajoute :

(Il bastone) non è solo l'immagine concreta del mezzo di sostegno del viandante, ma adombra un significato sacrale : è la verga usata con funzione divinatoria o magica sin dai tempi più antichi, e non solo presso i Greci ; ad Asclepio, quindi, viene riconosciuta la funzione iatromantica che già era del padre ${ }^{70}$.

À Cos, siège d'un sanctuaire très important et d'une ancienne école médicale, on célébrait le dieu avec l'analepsis tou rabdou: une procession solennelle, guidée par les Asclépiades, rejoignait le bosquet sacré des cyprès du dieu et lui donnait un nouveau bâton ${ }^{71}$. On en a une attestation unique en Ps.Hippocrate, Ep. 9 : le rituel devait être très ancien, car la ligue des Asclépiades disparut à la fin du IV siècle. D'après S.M.Sherwin-White il s'agissait d'un rite de passage, tandis que le bâton symbolisait le pouvoir de la divinité ${ }^{72}$.

66. Voir Chiara De Filippis Cappai, «Il culto di Asclepio...», 273. Témoignages anciens : Pausanias, 2,27,2 ; Eusèbe, P.E. 3,11,26 ; Eudocia, Aug. Viol. 11 ; Ovide, Mét. 15,655; Cornutus, Theol.Gr.Comp 33 (le bâton représente le salut); Arnobe, Adv.Nat. 6, 25.

67. Eduard Thrämer, « Asklepios », dans Wilhelm H. Roscher, Ausführliches Lexikon der Griechischen und Römischen Mythologie, vol. I, Leipzig, Teubner, 1884-86, 615-41 ; l'iconographie du serpent et du bâton est étudiée aux pages 628-629.

68. Giannina Solimano, Asclepio..., 141.

69. Kàroly Kerenyi, Asklepios..., cit. 54.

70. Chiara De Filippis Cappai, « Il culto di Asclepio... », cit. 274-275.

71. Voir Alice Walton, Asklepios..., 71-72 : selon elle, il s'agirait d'un acte symbolique de l'arrivée du dieu à Cos ou d'un de ses voyages ; Francesco Sirano, «Considerazioni... », 225. Pour Cos, Kàroly Kerenyi, Asklepios..., 47-69.

72. Voir Susan M. Sherwin-White, Ancient Cos. A Historical Study from the Dorian Settlement to the Imperial Period (Hypomnemata. Untersuchungen zur Antike und zu ihrem Nachleben 51), Göttingen, Vandenhoeck \& Ruprecht, 339-340 et 356-357. 
J. Schouten souligne le symbolisme de la guérison et de la résurrection, de l'énergie de la vie qui se dégage du monde végétal et de l'univers chthonien : pour cette raison, le bâton serait équivalent du serpent ${ }^{73}$. D'ailleurs, ce symbole est très riche et il est brandi constamment par magiciens et prêtres, comme expression de pouvoir, surtout surnaturel ${ }^{74}$.

Le reptile, en revanche, est l'animal qui manifeste par excellence le dieu guérisseur : "Indeed, the serpent and the god are alternate forms of one kind of epiphany $»^{75}$.

Il devait incarner l'aspect chthonien de cette divinité, reconnaissable aussi dans la pratique oniromantique, les rêves venant de la terre où sont enterrés les défunts; de plus, la terre donnait les principaux médicaments et les esprits aidaient le processus de guérison ${ }^{76}$. Dans

73. Jan Schouten, The Serpent of Asklepios..., 41-42.

74. Pour une histoire des connotations de ce symbole, voir Ferdinand Jozef M. De Waele, The Magic Staff or Rod in Graeco-Italian Antiquity, Erasmus, Gent, 1927.

75. Voir Howard Clark Kee, «Self-Definition in the Asclepius Cult... », cit. 123. Sources littéraires principales sur la connexion entre cet animal et le dieu: Hippocrate Ep. 15 ; Aristophane, Plutus 690 et-733-741 (les serpents sacrés lèchent les yeux de Ploutos); Héronde 4,91; Pausanias 2,27,2; ib. 2,28,1; ib. 9,39,3; Artémidore, 2,13; Élien, N.A. 8,12; Eusèbe, P.E. 3,11, 26 ; Pline NH 29, 72 ; Stace, Silv. 3, 4, 25 ; Macrobe, Saturn. 1, 20,1ss, etc.

76. Voir Alice Walton, Asklepios..., 8-11 et 16-17. Eduard Thrämer, «Asklepios », 1655 et 1681-82 croit fermement au caractère chthonien de cet attribut. Selon Christa Benedum, Asklepios - Der homerische Artz..., 214, ni le serpent, ni le bâton, n'apparaissent dans l'iconographie avant la fin du $\mathrm{V}^{\mathrm{e}}$ siècle, et, pour cette raison, il ne suffirait pas pour prouver le caractère chthonien du dieu. Des aspects typiques du culte chtonien, comme l'oniromantique, les incubations, le reptile, etc. seraient donc anciens, mais issus de la réorganisation du culte à Épidaure et étrangers à la nature primitivement héroïque d'Asclépios. Par contre, Alice Walton défend l'origine chthonienne de ce complexe d'éléments et explique que l'œuvre homérique, en tant que ionienne, n'était pas familiarisée avec ces croyances, plus diffuses dans la Grèce continentale; aussi, Homère aurait-il représenté Asclépios comme un simple héros. J'ajoute que la dense polysémie des images du serpent et du bâton et la richesse de la composante chthonienne fait songer, même en absence d'une documentation très ancienne, à un lien archaïque avec ce culte. $\mathrm{Au}$ demeurant, Christa Benedum n'explique pas l'origine du serpent et de l'incubation, traits chthoniens : même le lien qu'elle postule entre Asclépios et les déesses mères la dément (voir Christa Benedum, «Asklepiosmythos... », 55). 
les temples, le serpent était censé lécher les membres malades ${ }^{77}$; mais il se nourrissait aussi de rongeurs, ce qui assurait une meilleure hygiène aux établissements cultuels ${ }^{78}$. La fondation d'un nouveau sanctuaire impliquait souvent l'arrivée du serpent sacré ${ }^{79}$, et le prénom «Drako » apparaît couramment parmi les Asclépiades de Cos (même le fils d'Hippocrate s'appelait ainsi) ${ }^{80}$.

Le bâton avec un serpent enroulé apparaît constamment dans les statues du dieu ${ }^{81}$. Les témoignages écrits sont concordants : un

77. Voir Rudolf Herzog, «Die Wunderheilungen von Epidauros... », passim, qui illustre les Iamata d'Épidaure et nomme le serpent plusieurs fois comme protagoniste du culte. Selon cet auteur, le reptile et l'incubation sont des éléments indiscutables du culte chthonien.

78. Voir Kàroly Kerenyi, «On Snakes and Mice in the Cults of Apollo and Asklepios », dans Asclepios..., 102-107. Les zoologues ont identifié l'animal dans l'Elapha longissima, qui n'est pas venimeuse et habite les lieux des anciens temples du dieu: Gerald M. Hart, « Asclepius, God of Medicine », Canadian Medical Association Journal 92 (1965), 232-236. Herzog y voit par contre le Coluber longissimus, ou Coluber Aesculapii (voir Die Wunderheilungen von Epidauros..., 87-88, note 44). La tholos d'Epidaure pourrait avoir été un lieu d'ablutions ou le siège des serpents sacrés : Howard Clark Kee, "SelfDefinition in the Asclepius Cult... », 123 ; mais voir la critique de René Josef Ruettimann, Asclepius and Jesus..., 21-27.

79. Voir par exemple Giulia Sfameni Gasparro, «Alessandro di Abonutico, lo «pseudo-profeta » ovvero come costruirsi un'identità religiosa », Studi e Materiali di Storia delle Religioni 20 (1996), 565-590, surtout 571-572 (réproduit dans Id., Oracoli, profeti, sibille. Rivelazione e salvezza nel mondo antico, Rome, 2002, 149-202), qui mentionne Athènes, Sicion, Halieis, Épidaure Limera et Rome.

80. Voir Kàroly Kerenyi, Asklepios..., 55-56. Selon ce savant, le reptile aurait été le totem de la famille.

81. On peut en trouver un catalogue presque complet des types chez Bernard Holtzmann, «Asklepios », et Zlatozara Goceva, «Asklepios (in Thracia) », dans Lexikon Iconographicum Mythologiae Classicae, vol. II, 1 et II, 2 (photographies), Zurich-Munich, Artemis Verlag, 1984, 863-901 et 631-669 ; un bon panorama est offert aussi par Günther Heiderich, Asklepios (InauguralDissertation zur Erlagung der Doktorwürde der Philosophischen Fakultät der Albert-Ludwigs-Universität zu Freiburg in Breisgau), Giessen, Schreib-Druck, 1966 ; Eduard Thrämer, «Asklepios », 1690-97 ; Jan Schouten, Serpent of Asklepios..., 23-48. À 71-99 et 133-219, Schouten présente en outre une riche série d'exemples de la survivance du motif dans l'art médiéval et moderne : l'auteur démontre que le bâton d'Asclépios s'est progressivement émancipé de la figure du dieu, pour acquérir une vie iconographique propre, comme symbole de la médecine. Pour l'iconographie moderne, voir aussi Jane Davidson 
passage de Galien, un commentaire au serment hippocratique (dont on possède seulement la traduction arabe, faite à partir du syriaque) décrit le dieu avec son bâton à serpent ${ }^{82}$, peut-être en relation avec l'iconographie répandue dans les écoles médicales asiates du ${ }^{\mathrm{e}}{ }^{\mathrm{e}}$ siècle ${ }^{83}$. Les monnaies frappées dans les villes douées d'un Asclepieion répètent un motif identique ${ }^{84}$, qui était devenu l'emblème de $\operatorname{Cos}^{85}$. Même la fille du dieu, Hygieia, était couramment figurée en train de nourrir les serpents sacrés (Paus. I, 23, 5) ${ }^{86}$.

Enfin, il faut rappeler que le dieu était identifié aussi avec une constellation. Au ciel, Asclépios était le Serpentarius (Ophioukos; cf. Hygin, Astr. 14, 5) : cette constellation « soutient» la Couronne et se trouve à côté du Serpent. En fait, Serpentarius et Serpens sont vus souvent comme une formation unique, figurant le dieu Asclépios avec son animal ${ }^{87}$. Or, dans cette partie du ciel les étoiles ne disparaissent jamais complètement derrière l'horizon, raison pour laquelle leur lever se confond avec leur coucher : il s'agit du cercle arctique, fixé, dans l'antiquité, à $36^{\circ}$ du pôle. Sa position avait été fixée artificiellement par les astronomes comme unique pour la Grèce et l'Italie et correspondait à l'île de Rhodes, la patrie d'adoption d'Hipparque.

Reid, The Oxford Guide to Classical Mythology in the Arts, 1300-1990s, vol. I, New York-Oxford, Oxford University Press, 1993, 234-235. Pour des études plutôt focalisées sur des types nés ou répandus à l'époque considérée ici et dans la zone microasiatique, voir Francesco Sirano, « Considerazioni.... » ; Peter Kranz, «Die Asklepiosstatue im Schlosspark.... »; Kazimierz Michalowski, «Les deux Asclépios de Néa Paphos », Revue Archéologique 61 (1968), 355-358.

82. Voir Gotthard Strohmaier, «Asklepios und das Ei. Ikonographie in einem arabisch erhaltenen Kommentar zum hippokratischen Eid », dans R. Stiehl et H. E. Stier éd., Beiträge zur alten Geschichte und deren Nachleben : Festschrift für Franz Altheim zum 6.10.1968, vol. II, Berlin, De Gruyter, 1970, 143-153, surtout 146-147.

83. Francesco Sirano, « Considerazioni... », 224.

84. Jan Schouten, The serpent of Asklepios..., 33 et, surtout, Peter Robert Franke, «Asklepios - Aesculapius... »; des nombreuses monnaies remontent $\mathrm{au} \mathrm{II}^{\mathrm{e}}$ siècle et à la région asiate.

85. Voir Rudolf Herzog, « Die Wunderheilungen von Epidauros... », 147148.

86. Gioia De Luca, «Asklepios in Pergamon », dans Bernard Andreae et alii éd., Phyromachos Probleme..., 25-40 ; Kàroly Kerenyi, Asklepios..., passim et Peter Robert Franke, «Asklepios - Aesculapius... », passim.

87. Wilhelm Gundel, «Ophis », dans RE 18, 1, 649-654. 
Selon Strabon, 2,1,1 (qui cite, peut-être, Eudoxe) cette latitude coupe le monde en deux moitiés ${ }^{88}$.

En revanche, Aristide, 4,56, décrit une apparition du dieu en rêve, où il se manifeste au centre du ciel, à l'instar de l'Anima mundi. Il existait en fait une tradition philosophique, qui avait conduit à une interprétation médio-platonicienne d'Asclépios comme anima mundi. G. Sfameni Gasparro commente ainsi le texte d'Aristide ${ }^{89}$ :

L'Asclepio pergameno, dio medico e oracolare, cui i fedeli di ogni ceto sociale si rivolgevano per ottenere soprattutto la guarigione dalle malattie e comunque garanzie per l'esistenza terrena, è così recuperato in una vasta prospettiva teologica di marca platonica, proponendosi la sua identificazione con il principio animatore del grande Tutto.

Cela nous ramène finalement aux Pérates. En effet, d'après la notice de $E l .5,16,15-16$, en regardant le ciel on pourra voir la « belle image du serpent», qui serait le «commencement de tout mouvement pour tous les êtres »; c'est pourquoi, tout existerait grâce à lui. Cela laisse entendre sa fonction d'anima mundi. Près de sa tête, de plus, on pourrait observer l'union du lever et du coucher. À ce moment, le rédacteur de la notice emploie des mots provenant « de l'ignorance », c'est-à-dire, de la sagesse païenne, et il cite les vers d'Aratos concernant le Draco et le groupe des constellations autour du Serpens et du Serpentarius (cf. Ar. 40-85). Selon les Pérates, le Logos adoré par le groupe, le serpent, est visible aussi sous la forme du Draco dans le ciel : mais l'antiquité le confondait souvent avec le serpent de la constellation qui équivaut à Asclépios. Cela va dans le même sens que le témoignage fourni par Aristide : Asclépios peut être considéré comme l'anima mundi, le principe animateur du Tout de la pensée médio-platonicienne. La théologie des Apologistes avait donné ce rôle au Fils, qui transmettait ainsi la pensée du Père au kosmos, en accomplissant sa propre fonction créatrice ${ }^{90}$.

88. Cf. aussi Ératosthène, Catast. 1,6; Servius, Comm.in Aen. 9, 259.

89. Giulia Sfameni Gasparro, « Oracolo, divinazione, profetismo nel mondo greco-romano da Augusto alla fine del II sec. », Ricerche Storico-Bibliche 5 (1993), 11-42, cit. 36.

90. Voir Jean Daniélou, Message évangélique et culture hellénistique aux $I I^{e}$ et $I I I^{e}$ siècles, Paris, Desclée/Cerf, 1961, 317-353. 
Un passage en particulier confirme cette perspective par le recours à l'image du serpent d'airain : il s'agit de Justin, Apol. 60. Dans la conception du philosophe chrétien, le Christ est l'anima mundi, Fils de Dieu, qui se manifeste, selon la vision platonicienne, dans le $\mathrm{X}$ qui traverse le ciel (Platon, Tim.36b-c). Pour expliquer ce concept au moyen des Écritures, Justin relate l'histoire du serpent d'airain ; il ne mentionne pas cependant le serpent, hissé sur la hampe, mais un générique typos staurou, une « image de la croix ». On peut supposer que cette négligence est voulue et cache le rejet d'une interprétation qui pouvait aller dans le sens du syncrétisme et d'un syncrétisme que Justin connaissait vraisemblablement très bien, car il avait vécu un certain temps à Éphèse : cela signifie que le serpent, notamment celui d'Asclépios, pouvait être considéré comme anima mundi. Il ne faut pas oublier non plus que les Pérates utilisent des testimonia analogues à ceux de Justin.

On doit donc en venir maintenant à l'étonnante coïncidence entre le bâton d'Asclépios et le serpent d'airain : une coïncidence double, de forme (car les deux symboles sont représentés de la même façon) et de contenu (car les deux sont des serpents guérisseurs). Il ne faut pas une grande fantaisie pour se rendre compte que le serpent d'Asclépios, enroulé autour de son bâton, est iconographiquement identique au serpent d'airain biblique, type, selon l'Évangile de Jean, du Christ élevé et crucifié. J. Schouten avait déjà remarqué cette identité il y a plusieurs années ${ }^{91}$. Pour les Pérates, le serpent d'airain est au cœur de leur dossier exégétique : il est le Logos ; il est identique à la constellation d'Asclépios et au symbole iconographique de cette divinité, qui, à son tour, est considérée comme le Logos, anima mundi, en accord avec une ligne de pensée très vivante parmi les Apologistes et dans le médio-platonisme ${ }^{92}$. Bref, il est vraisemblable que les Pérates aient conçu une forme de syncrétisme entre le christianisme et le culte de ce dieu grec, origine de l'ophitisme.

Mais le lien entre serpent d'airain et cultes de guérison pourrait être bien plus ancien. Vernes en a repéré les traces à l'origine du passage vétéro-testamentaire de $N b$. 21, 8-10 : peut-être les Hébreux

91. Jan Schouten, The Serpent of Asklepios..., 99-106.

92. Jean Daniélou, Message évangélique et culture hellénistique..., loc. cit. 
entrèrent-ils en contact avec un sanctuaire d'Eshmoun, un épisode que la version biblique essaya de légitimer en attribuant la construction de l'objet à l'initiative divine et de Moïse ${ }^{93}$. On aurait donc ici une première trace d'identification entre Asclépios (= le dieu Eshmoun) et Yahvé ${ }^{94}$. En fait, selon une recherche récente, l'unique véritable dieu guérisseur de la zone sémitique occidentale était justement Yahvé, car aucune autre divinité ne possédait ces qualités d'une façon ainsi forte; c'est seulement à l'époque hellénistique que le syncrétisme entre Asclépios et Eshmoun aurait pris la relève ${ }^{95}$. I. Benzinger mentionne l'association traditionnelle entre serpent et eau, surtout eau sacrée et guérisseuse, sans oublier sa connexion avec l'au-delà, la sagesse et la divination: dans son ouvrage, il montre le dessin d'un petit serpent d'airain, retrouvé dans le sanctuaire de Gezer. Il a donc lancé l'hypothèse que le Nehustan, le serpent d'airain gardé dans le temple de Jérusalem jusqu'à sa destruction par ordre du roi Ezéchias, était considéré comme rien moins qu'une représentation de Yahvé ${ }^{96}$. Hypothèse plausible : le déni exprimé par Vernes, qui décrit le Nehustan comme une simple « relique », pour laquelle les Hébreux auraient brûlé de l'encens en simple et anodin acte d'hommage, ne semble guère convaincant.

Les Pérates avaient donc identifié le Christ à Asclépios, vraisemblablement considéré comme son incarnation païenne ante litteram. Les deux divinités possédaient des attributs presque identiques, leur activité était analogue et même leur vie sur terre comportait des

93. Maurice Vernes, « Le serpent d'airain fabriqué par Moïse et les serpents guérisseurs d'Esculape », Revue Archéologique 7 (1918), 36-49.

94. L'identification est ancienne comme le montre l'inscription trilingue CIS I, 143 (en latin, grec et punique) ; elle devint plus courante à l'époque hellénistique. Voir aussi Eduard Thrämer, «Asklepios », 1679-1680.

95. Voir Micheal L. Brown, « Was there a West Semitic Asclepios? », UgaritForschungen 30 (1998), 133-154. Le syncrétisme entre les deux divinités, l'une grecque, l'autre phénicienne, est illustré par une monnaie de 145-147, en l'honneur de Marc-Aurèle, alors prince héritier, où les serpents dressés à côté du trône sont un attribut iconographique d'Eshmoun : voir Peter Robert Franke, «Asklepios - Aesculapius auf antiken Münzen... », 67.

96. En tout cas, il propose aussi l'identification alternative avec la divinité féminine Ishtar: Immanuel Benzinger, Hebräische Archäologie, Leipzig, E.Pfeiffer Verlag, $1927^{3}, 327$. 
analogies. Pour un païen récemment converti, un syncrétisme de ce type n'aurait rien eu de suspect. En outre, l'insistance des Pérates sur les testimonia ligni et crucis trouve son explication naturelle dans la fusion syncrétiste qu'ils ont opérée entre la croix du Christ et le bâton d'Asclépios : il n'est pas hors de propos de rappeler que même l'iconographie des miracles évangéliques attribue la virga thaumaturgica à Jésus sur les sarcophages du IV siècle, comme dans la scène de la résurrection de Lazare sculptée sur un sarcophage exposé aux Musées Capitolins ${ }^{97}$. On peut donc clore le débat sur la vexata quaestio du rapprochement entre le Christ et Asclépios : celui-ci existait et était dangereux. Enfin, on peut confirmer une datation de la secte des Pérates au milieu du $\mathrm{II}^{\mathrm{e}}$ siècle et supposer raisonnablement son enracinement dans l'environnement asiate.

\section{SABAziUs ?}

Avant de conclure, j'aimerais ajouter de brèves références à d'autres cultes, très répandus au $\mathrm{II}^{\mathrm{e}}$ siècle et qui témoignent de la renommée du serpent à cette époque : celui du dieu Sabazius et celui introduit par Alexandre d'Abounotichos pour le serpent Glykon. Commençons par Sabazius.

D'origine thrace ou anatolienne, Sabazius était connu dans toute la Méditerranée ${ }^{98}$. Rapidement associé aux mystères de Cybèle ${ }^{99}$,

97. Voir Erich Dinkler, Christus und Asklepios..., 13, 35 et Ferdinand Jozef M. De Waele, The Magic Staff or Rod....

98. Voir Sherman E. Johnson, « The Present State of Sabazios Research », dans ANRW II, 17, 3, 1583-1613 (avec une riche bibliographie) ; Charles Picard, « Sabazios, dieu thraco-phrygien: expansion et aspects nouveaux de son culte », Revue Archéologique 54 (1961), 129-176. Theodossiev a récemment proposé une étymologie thrace du nom, qui témoignerait de l'union du dieu solaire paternel avec la déesse mère-montagne, union typique dans ce milieu religieux : Nikola Theodossiev, «Semantic Notes on the Theonyms Orpheus, Sabazios and Salmoxis », Beiträge zur Namenforschung 29/30 (1994/95), 241-246. Selon Isabelle Tassignon, ce dieu tirerait son origine du mythe hittite d'Illuyanka, le serpent-dragon vaincu par le dieu de la tempête: Isabelle Tassignon, « Sabazios, dans le panthéon des cités d'Asie Mineure », Kernos 11 (1999), 189-208.

99. Voir Charles Picard, «Sabazios, dieu thraco-phrygien... », 159. 
il subit de fréquentes contaminations avec des rituels de caractère extatique et liés à la fertilité ; en Asie Mineure, en plus, on gardait aussi la connexion avec le niveau céleste, ce qui permit son identification progressive avec Zeus (depuis le $\mathrm{II}^{\mathrm{e}}$ siècle a.c. au moins). Ce dieu était très populaire parmi les esclaves, souvent d'origine asiatique $^{100}$ : à l'époque impériale, sa renommée sera largement diffusée par les soldats, surtout en Dacie.

Les sources apportent de nombreux éléments sur l'importance du serpent dans ce culte, où il aurait incarné le dieu même ${ }^{101}$. Les auteurs chrétiens soulignent qu'on laissait glisser le serpent sur le corps des initiés : il s'agirait là, selon plusieurs savants, d'un mariage sacré. Démosthène parle aussi avec dégoût des serpents maniés dans le culte (cf. De corona, 260). La valeur chthonienne est très probable, car elle est confirmée par l'autre attribut du dieu, la pomme de pin $^{102}$. Parmi les objets cultuels, on comptait aussi la kyste contenant un reptile ${ }^{103}$. Diodore, 4, 4, explique cette présence de l'animal grâce au mythe : Zeus aurait engendré sous forme de reptile le dieu Dionysos-Zagreus-Sabazius de Koré ; mais ce mythe laisse apercevoir clairement une rationalisation postérieure. Enfin, le serpent domine dans l'iconographie du dieu ${ }^{104}$ : il apparaît dans les inscriptions qui lui sont dédiées; dans deux cas, l'animal entoure un arbre ${ }^{105}$.

100. Sherman E. Johnson, « The Present State of Sabazios Research... », 1588-1590 ; Charles Picard, « Sabazios, dieu thraco-phrygien... ».

101. Cf. Clément d'Alexandrie, Protr. 2, 16, 2 et Arnobe, Adv.Nat. 5, 20-21.

102. Voir Youssef Hajjar, « À propos d'une main de Sabazios au Louvre », dans Margreet B. de Boer et alii éd., Hommages à Maarten J.Vermaseren. Recueil d'études offert par les auteurs de la Série Études préliminaires aux religions orientales dans l'Empire Romain à Maarten J. Vermaseren à l'occasion de son soixantième anniversaire le 7 Avril 1978 (Études préliminaires aux religions orientales dans l'Empire Romain 68), vol. I, Leyde, Brill, 1978, 455-472, surtout 460-461.

103. Sherman E. Johnson, « The Present State of Sabazios Research... », 1587.

104. Charles Picard, «Sabazios, dieu thraco-phrygien... », passim.

105. Voir Eugene N. Lane, « Towards a Definition of the Iconography of Sabazius », Numen 27 (1980), 9-33. 
Très connues sont les mains du dieu, ornées de différents objets, parmi lesquels le serpent est toujours présent ${ }^{106}$.

De plus, ces mystères ont été maintes fois soupçonnés d'avoir encouragé le syncrétisme avec le judaïsme ${ }^{107}$. Johnson soulève de nombreux doutes à ce sujet, en affirmant que les sources n'offrent pas de données sûres et même que des païens pouvaient avoir identifié Sabazius avec Yahvé, à cause de l'étymologie populaire Sabazius = Sabaoth. Certaines sources classiques semblent confirmer que le syncrétisme provenait plutôt d'une ambiance païenne ${ }^{108}$. Johnson a probablement raison sur le fond, en niant l'existence d'un phénomène de syncrétisme juif très élargi et structuré ; mais il est difficile d'éliminer totalement cette possibilité de l'histoire des religions d'époque impériale, notamment parce que les premières communautés chrétiennes, d'origine juive (et de la Diaspora), ont vu fleurir chez elles le syncrétisme, ce qui est montré par certains groupes gnostiques. A. Duprez rappelle que l'orthodoxie pharisienne était bien loin de l'usage religieux des masses palestiniennes ; il donne aussi l'exemple du temple d'Éléphantine, pour rappeler que le judaïsme n'était pas monolithique ${ }^{109}$. En outre, L. W. Barnard décrit le judaïsme de la Diaspora comme hellénisé et proche des croyances ésotériques, pour ne pas dire syncrétistes ${ }^{110}$. Je suis donc d'accord avec Johnson, pour reconnaître que le syncrétisme devait être surtout un produit

106. Sherman E. Jonhson, « The Present State of Sabazios Research... », 1591-98. Ces mains expriment la puissance protectrice du dieu, voir Youssef Hajjar, « À propos d'une main de Sabazios... »; Eugene N. Lane, « Towards a Definition... », 12-16.

107. On a souvent invoqué comme soutien le passage de Valère Maxime 1,32, où, pourtant, le mot Iudaeos semble une glose : Eugene N. Lane, «Sabazius and The Jews in Valerius Maximus: A Re-Examination », Journal of Romen Studies 69 (1979), 35-38. Cela affaiblit la construction de Franz Cumont, «Les mystères de Sabazius et le judaïsme », Comptes Rendus de l'Académie des Inscriptions et Belles-Lettres 1906, 63-97 ; Id., « À propos de Sabazius et le judaïsme », Le musée belge 14 (1910), 55-60.

108. Cf. Plutarque, Quaest. Conv. 4, 6, Tacite, Hist. 5, 4-5.

109. Antoine Duprez, Jésus et les dieux guérisseurs..., 122.

110. Leslie W. Barnard, « The Old Testament and Judaism in The Writings of Justin Martyr », Vetus Testamentum 14 (1964), 395-406. 
païen, mais je serais plus prudente par rapport à son absence complète du judaïsme, surtout celui de la Diaspora.

En tout cas, l'époque impériale a connu un autre culte focalisé sur le serpent, celui de Sabazius. Même si les Pérates proviennent d'une fusion entre christianisme et culte d'Asclépios, la proximité géographique et temporelle (c'est-à-dire la renommée des mystères de Sabazius en Asie Mineure au II $^{\mathrm{e}}$ siècle) permet de supposer que ce culte a pu également avoir une influence sur l'importance centrale donnée par l'ophitisme à la figure du serpent : la possibilité d'un syncrétisme entre paganisme et judaïsme renforce cette hypothèse. On peut aussi remarquer qu'il existe des exemples de rapprochement entre les deux cultes, comme le souligne I. Tassignon ${ }^{111}$.

\section{ALEXANDRe D'Abounutichos ET LE CULTE DU SERPENT GLYKon}

Les célèbres mystères de Glykon inventés par Alexandre d'Abounutichos au milieu du $\mathrm{II}^{\mathrm{e}}$ siècle, une véritable apothéose du serpent, se présentent comme une filiation du culte d'Asclépios ${ }^{112}$. Comme on le sait, le prophète Alexandre avait su créer un culte nouveau en exploitant les attentes religieuses de ses concitoyens : il avait fondé dans sa ville natale d'Abounotichos un oracle, qui ne négligeait pas non plus l'aspect guérisseur. D'une façon unique le

111. I. Tassignon («Sabazios dans le panthéon... ») 193, note 22. La validité de l'observation n'est pas affaiblie du fait que, selon l'auteur, il s'agit d'un phénomène tardif.

112. Il est bien connu que la source principale sur ce culte est l'opuscule de Lucien, Pseudomantis : pour une édition, voir Marcel Caster-Pierre-Emmanuel Dauzat éd., Alexandre ou le faux prophète (Classiques en poche 46), Paris, Belles Lettres, 2001. Sur les limites à accorder au témoignage de Lucien, Graham Anderson, «Lucian: Tradition versus Reality », ANRW II, 34, 2, 1422-1447 ; sur Alexandre d'Abounothicos voir 1435-1437 ; aussi Diskin Clay, «Lucian of Samosata: Four Philosophical Lives (Nigrinus, Demonax, Peregrinus, Alexander Pseudomantis) », dans ANRW II, 36, 5, 3406-3450. Le scepticisme du rhéteur était dû à son lien avec l'épicurisme : François Guillaumont, « Lucien et la divination », dans Les écrivains du deuxième siècle et l'Etrusca disciplina. Actes de la Table Ronde de Dijon 9 juin 1995. La divination dans le monde étrusco-italique VII (Caesarodunum Suppl. 65), Université de Tours, 1996, 13-25. 
« dieu », le serpent Glykon, était physiquement présent dans le temple et donnait même des réponses par sa propre « voix » ${ }^{113}$. Ce culte connut un succès incroyable dans tout l'Empire, avec des manifestations de faveur de l'empereur Marc-Aurèle lui-même. Si l'iconographie du serpent Glykon, loin de celle d'Asclépios et montrant un serpent barbu ${ }^{114}$, nous empêche de voir en ces mystères une source directe d'inspiration pour les Pérates, ce culte est néanmoins témoin de la faveur énorme dont cet animal jouissait dans la seconde moitié du II $^{\mathrm{e}}$ siècle.

113. Flinterman a fixé récemment la date du voyage de Lucien à Abounotichos à la fin de l'été 161-162 : voir Jaap-Jan Flinterman, « The Date of Lucian's Visit to Abunoteichos », Zeitschrift für Papyrologie und Epygraphik 119 (1997), 280-282. Pour la chronologie du phénomène, Auguste V. B. Miron, «Alexander von Abonuteichos. Zur Geschichte des Orakels des Neon Asklepios Glykon », in Wolfgang Leschhorn-Auguste V. B. Miron-Andrei Miron éd., Hellas und der griechische Osten. Studien zur Geschichte und Numismatik der griechischen Welt. Festschrift für Peter Robert Franke zum 70. Geburtstag, Saarbrücken, Saarbrücker Druckerei, 1996, 153-188. Un document important, remontant à la fin du II ${ }^{\mathrm{e}}$ siècle, est l'autel décrit par Walter Ameling, «Ein Altar für Alexander von Abunoteichos », Epigraphica Anatolica 6 (1985), 34-36. Sur Alexandre et le culte du serpent Glykon, Giulia Sfameni Gasparro, « Alessandro di Abonutico, lo "pseudo-profeta" ovvero come costruirsi un'identità religiosa », Studi e Materiali di Storia delle Religioni 20 (1996), 565-590, surtout 565 ; Giulia Sfameni Gasparro, « Alessandro di Abonutico, lo "pseudo-profeta" ovvero come costruirsi un'identità religiosa. II. L'oracolo e i misteri », dans Corinne Bonnet éd., Les syncrétismes religieux dans le monde méditerranéen antique. Actes du Colloque International en l'honneur de Franz Cumont à l'occasion du cinquantième anniversaire de sa mort. Rome, Academia Belgica, 25-27 septembre 1997, Bruxelles-Rome, Institut Historique Belge de Rome, 1999, 275-305 ; Giulia Sfameni Gasparro, « Oracolo, divinazione, profetismo nel mondo grecoRomano da Augusto alla fine del II sec. », Ricerche Storico-bibliche 5 (1993), 11-42 (tous trois reproduits dans Id., Oracoli, profeti e sibille..., 149-202 pour les deux premiers, 23-60 pour le dernier). Sur les artifices opérés par Alexandre pour feindre les oracles « autophones », voir Renée Piettre, «Un buste parlant d'Epicure. La philosophie au péril des religions », Kernos 15 (2002), 131-44. À propos du développement de la superstition au II $^{\mathrm{e}}$ siècle, surtout en lien avec l'épidémie de 165-180, Christa Benedum, «Betrachtungen zu Asklepios und dem Aesculapius der Römer.... », 200.

114. Pour l'iconographie de Glykon, voir Gabriella Bordenache Battaglia, «Glykon", dans Lexikon Iconographicum Mythologiae Classicae, vol. 4/1, Zurich-Munich, Artemis, 1988, 279-283 ; Auguste V. B. Miron, «Alexander von Abonuteichos...» (numismatique). 


\section{Conclusions}

On a vu la grande importance accordée au serpent au $\mathrm{II}^{\mathrm{e}}$ siècle : image d'immortalité et de salut, il apparaît presque partout et récupère, dans la conscience collective, le rôle qu'il avait eu dans la religion chthonienne archaïque ; il est aussi identifié avec le dieu suprême. Au II ${ }^{\mathrm{e}}$ siècle, qui dit serpent, dit Asclépios : c'est surtout ce culte qui met le reptile «à la mode », sans oublier les autres cultes à mystères, notamment de Sabazius et de Glykon.

Dans ce cadre, il ne faut pas s'étonner si quelqu'un a songé à mêler Jésus avec le serpent : il faudrait plutôt s'étonner du contraire. J'ajouterai que, si l'ophitisme n'était pas né au II' siècle, il n'aurait plus eu d'occasion aussi propice pour apparaître. D'après J.-D. Kaestli, mais aussi B. A. Pearson, on peut supposer que les Pérates furent les véritables fondateurs de l'ophitisme au milieu du II $^{\mathrm{e}}$ siècle. En fait, le passage de $N b .21,8-10$, déjà employé par $J n .3,14$, est primordial pour comprendre comment certains gnostiques ont pu identifier Jésus avec le serpent et adorer celui-ci. Cette identification est au cœur de l'enseignement des Pérates; leur pensée recueille l'équation Logos = anima mundi des Apologistes et du médioplatonisme. Pour les fidèles du dieu de Pergame, comme Aristide, on pouvait établir aussi l'équation Asclépios = anima mundi . En professant l'identité entre serpent d'airain et serpent d'Asclépios, les Pérates créèrent donc un lien syncrétiste entre les deux : leur action se comprend très bien sur le fond des cultes de salut de cette époque.

Cette connexion avec Asclépios permet aussi une autre hypothèse vraisemblable : les Pérates (et donc les Ophites) pourraient avoir vu le jour dans le milieu asiate, qui témoigne de la grande importance d'Asclépios, mais aussi de la progressive diffusion du christianisme, surtout de matrice johannique. Cela expliquerait aussi pourquoi Épiphane fait remonter les Ophites aux Nicolaïtes (cf. Ap. 2, 6-15). En même temps, nous pouvons confirmer le rôle du christianisme dans la naissance de quelques groupes gnostiques. Ceux-ci, en quête du Sauveur, pensèrent l'avoir trouvé dans l'animal symbolisant le salut, exalté par Moïse au désert et déjà présent parmi les 
païens comme attribut d'un dieu puissant et doux, très semblable au Christ : un Dieu qui daignait descendre sur terre, pour arracher ses fidèles au mal ${ }^{115}$.

Département de Patristique

Faculté de Théologie

Université de Fribourg

Suisse

115. J'aimerais adresser mes plus vifs remerciements au professeur Alain Le Boulluec, de Paris, mon directeur de thèse, ainsi qu'aux docteurs Jacques Rime et Jean-Michel Roessli, de Fribourg, pour leurs suggestions et pour leur précieuse révision de la langue française de cet article. 